\title{
Shape optimized inclined single and double wall wave barriers for ground vibration mitigation
}

\author{
J.D.R. Bordón ${ }^{\mathrm{a}, *}$, C. Van hoorickx ${ }^{\mathrm{b}}$, J.J. Aznárez ${ }^{\mathrm{a}}$, M. Schevenels ${ }^{\mathrm{c}}$, O. Maeso ${ }^{\mathrm{a}}$, G. \\ Lombaert $^{\mathrm{b}}$ \\ ${ }^{a}$ Universidad de Las Palmas de Gran Canaria, Instituto Universitario SIANI, Edificio Central del Parque \\ Cientifico y Tecnológico del Campus Universitario de Tafira, 35017 Las Palmas de Gran Canaria, Spain \\ ${ }^{b}$ KU Leuven, Department of Civil Engineering, Structural Mechanics Section, Kasteelpark Arenberg 40, \\ 3001 Leuven, Belgium \\ ${ }^{c}$ KU Leuven, Department of Architecture, Architectural Engineering, Kasteelpark Arenberg 1, 3001 \\ Leuven, Belgium
}

\begin{abstract}
Stiff wave barriers are capable of reducing the transmission of ground vibrations. Most designs consist of a single vertical wall, although double walls are also being considered. This paper investigates the shape optimization (position, inclination, length and thickness) of these topologies in a two-dimensional setting, for a point source and a point receiver placed symmetrically with respect to the design domain. Three types of sources are studied: a single-frequency source, a broadband source and a harmonic source within a given frequency range. An economical constraint on the maximum material use is considered. A multi-region BEM methodology is used for evaluating the objective function and its gradient. Analytical expressions are presented for the sensitivities, providing a very effective simulation tool for this type of problem. It is found that significant improvement can be achieved by repositioning and inclining the walls when compared to the reference cases. It is also found that optimized double wall barriers outperform single wall barriers. The improvement is insignificant for sources which generate Rayleigh wavelengths similar to the design domain depth, but it greatly increases as frequency increases and the penetration depth decreases.
\end{abstract}

Keywords: wave barrier, shape optimization, boundary element method, shape sensitivity, analytical sensitivity

\footnotetext{
${ }^{*}$ Corresponding author

Email addresses: jacobdavid.rodriguezbordon@ulpgc.es (J.D.R. Bordón), cedric.vanhoorickx@kuleuven.be (C. Van hoorickx), juanjose.aznarez@ulpgc.es (J.J. Aznárez), mattias.schevenels@kuleuven.be (M. Schevenels), orlando.maeso@ulpgc.es (O. Maeso), geert.lombaert@kuleuven.be (G. Lombaert) Postprint submitted to Soil Dynamics and Earthquake Engineering Published version: J.D.R. Bordón, C. Van hoorickx, J.J. Aznárez, M. Schevenels, O. Maeso, and G. Lombaert. Shape optimized inclined single and double wall wave barriers for ground vibration mitigation. Soil Dynamics and Earthquake Engineering, 112:215-231, 2018. https://doi.org/10.1016/j.soildyn.2018.04.035
} 


\section{Introduction}

Machinery and transportation systems are sources of vibrations that can travel through the soil to nearby constructions, where they can annoy people or cause equipment malfunctioning or even mechanical damage [1]. Whole-body vibrations are perceived in the frequency range $1-80 \mathrm{~Hz}$ [2], while higher frequency vibrations in the range $16-250 \mathrm{~Hz}$ lead to re-radiated noise inside buildings, which is also known as ground-borne noise [3]. In order to reduce these vibrations, a wave barrier can be installed along the transmission path as a passive attenuation system. For surface waves, open trenches are the best solutions to such problem since their stress-free boundaries act as perfect reflectors of elastic waves [4]. Their effectiveness greatly depends on the ratio between the Rayleigh wavelength and the trench depth. However, a pure open trench can not be excavated to any desired depth for soil stability reasons and the possible presence of ground water. Therefore, alternative systems such as open trenches reinforced with retaining sheet piles or concrete walls [5], in-filled trenches with soft [6] or stiff materials [7, 8], or the installation of sheet piles [9] or rows of piles [10], are also used. Nowadays, the versatility of construction methods such as jet grouting opens up the door for more complex designs based on in-filled trenches with stiff materials. Recently, the use of manufactured structured media (metamaterials/metabarriers) [11] is also being considered for guiding of Rayleigh waves.

Van hoorickx et al. [12] explored novel stiff wave barriers designs obtained via topology optimization [13]. The designs emerging from topology optimization greatly improve the performance of any other conventional wave barrier. They are capable of producing a very high insertion loss at target frequencies, or a considerably improved insertion loss within a frequency range. They are, however, quite complex and thus require manual post-processing in order to define a viable design. In the present paper, we study the problem from a different perspective by exploring the possibilities of the shape optimization of simpler feasible designs: single and double wall barriers; where position, inclination, length and thickness of walls are taken as design variables. Single vertical walls have been extensively studied, while double wall barriers have been considered recently [14]. The effect of wall inclination has been considered by Andersen et al. $[15,16]$, where it was observed that it is capable of improving barrier performance.

The Boundary Element Method (BEM) is probably one of the most used analysis 
tools for this type of wave propagation problems, and it is also used in this work. Open and in-filled trenches have extensively been studied through two-dimensional BEM models, see e.g. [17, 18]. Three-dimensional open and in-filled trenches and piles have also been studied $[19,20,10]$. Coupled BEM-FEM models are often used in order to incorporate structural members in a more natural and efficient manner $[6,8,9,21]$. Three-dimensional and two-and-a-half-dimensional models offer more realistic results at the expense of more computational costs. However, as argued by Andersen et al. [22], two-dimensional models lead to similar wave patterns, and they offer a good trade-off for most typical long buried structures. Therefore, for long wave barriers, a two-dimensional methodology offers a good compromise between reproducing the actual physical problem and having a moderate computational cost. This is especially true for optimization problems, where a number of designs are evaluated in an iterative procedure.

In the present work, gradient-based shape optimization is used. The use of the BEM in the context of gradient-based design optimization began in the 1980s for twodimensional heat conduction $[23,24]$ and elastostatics $[25,26]$ problems. More recently, Bonnet [27, 28, 29, 30] covered a wide range of shape sensitivity analysis using the BEM and a rigorous mathematical treatment. In particular, Bonnet [31] proved that material differentiation formulas for regular integrals still hold for strongly singular and hypersingular integrals, which demonstrated that material differentiation can be applied to non-regularized as well as regularized BIEs. Gallego, Rus and Suárez $[32,33,34,35,36]$ used the BEM for cavities and crack identification in potential and elastic problems, where geometric sensitivity BIEs derived from the Taylor's expansion of the shape perturbation are developed. In the present work, the latter technique is used to formulate the Geometric Sensitivity BEM, which is used in a direct approach rather than in an adjoint approach since a relatively small number of design variables is present in the studied problems.

The paper is organized as follows. In Section 2, the methodology and the formulation of the optimization problem are described. In particular, Sections 2.1 and 2.2 describe the use of the BEM for zero- and first-order geometric sensitivity analyses of time harmonic elastodynamics. Section 2.3 states the optimization problem and explains how it is solved. In Section 3, the methodology is validated, and then the obtained optimized wave barriers are described and results are interpreted from a physical 
point of view. Finally, conclusions are given in Section 4.

\section{Methodology}

\subsection{Geometric Sensitivity BEM for elastodynamics}

Let $\Omega$ be a region in $\mathbb{R}^{2}$ with boundary $\Gamma=\partial \Omega$ whose orientation is defined by the outward unit normal vector $\mathbf{n}=\left(n_{1}, n_{2}\right)^{T}$. Region $\Omega$ is an elastic solid under a plane strain state whose properties are: density $\rho$, Poisson's ratio $\nu$, shear modulus $\mu$, and Lamé's first parameter $\lambda=2 \mu v /(1-2 v)$. A hysteretic damping ratio $\xi$ can be considered by using complex elastic constants $\mu=\operatorname{Re}(\mu)(1+i 2 \xi)$ and $\lambda=\operatorname{Re}(\lambda)(1+i 2 \xi)$. Displacements are denoted as $u_{k}$, and tractions as $t_{k}=\sigma_{k j} n_{j}$, where the stress tensor is $\sigma_{k j}=\lambda u_{m, m} \delta_{k j}+\mu\left(u_{k, j}+u_{j, k}\right)$, and $k, j, m=1,2$. For the time harmonic analysis at circular frequency $\omega=2 \pi f$, the Singular BIE (SBIE) for an interior or boundary collocation point $\mathbf{x}^{\mathrm{i}}$ can be written as [37]:

$$
c_{l k}^{\mathrm{i}} u_{k}^{\mathrm{i}}+\int_{\Gamma} t_{l k}^{*} u_{k} \mathrm{~d} \Gamma=\int_{\Gamma} u_{l k}^{*} t_{k} \mathrm{~d} \Gamma
$$

where the body loads have been disregarded, $l=1,2$ is the live index related to the load direction, and $k=1,2$ is the dummy index related to the observation direction. In the present work, the superscript $\mathrm{i}$ is used to denote variables or parameters defined at the collocation point. Fundamental solutions in terms of displacements $u_{l k}^{*}$ and tractions $t_{l k}^{*}$ can be found elsewhere, e.g. [37]. The free-term $c_{l k}^{\mathrm{i}}$ for two-dimensional elastic problems can be found for example in [38]. The left hand side integral of Eq. (1) must be understood in the Cauchy Principal Value sense when $\mathbf{x}^{\mathrm{i}} \in \Gamma$. Assuming a discretization of $\Gamma$ based on isoparametric Lagrange elements with shape functions $\phi_{p}^{(e)}$, each boundary element $e$ introduces the following approximation:

$$
x_{k}=\phi_{p}^{(e)} x_{k p}^{(e)}, u_{k}=\phi_{p}^{(e)} u_{k p}^{(e)}, t_{k}=\phi_{p}^{(e)} t_{k p}^{(e)}
$$

where $p=1, \ldots, N_{\mathrm{n}}^{(e)}$, and $N_{\mathrm{n}}^{(e)}$ is the number of nodes of boundary element $e$. An appropriate collocation of Eq. (1) once discretized leads to the well-known relationship:

$$
\mathbf{H u}=\mathbf{G t}
$$


and, after applying boundary conditions, can be written as:

$$
\mathbf{A x}=\mathbf{B} \breve{\mathbf{x}}=\mathbf{b}
$$

where $\mathbf{A}$ is composed of components of the influence matrices $\mathbf{H}$ and $\mathbf{G}$ related to the unknown components of $\mathbf{u}$ and $\mathbf{t}$ (gathered in $\mathbf{x}$ ), and $\mathbf{B}$ is composed of components of $\mathbf{H}$ and $\mathbf{G}$ related to the known components (boundary conditions) of $\mathbf{u}$ and $\mathbf{t}$ (gathered in $\breve{\mathbf{x}})$. The solution of Eq. (4) provides displacements and tractions along $\Gamma$. Afterwards, Eq. (1) can be used to calculate displacements at internal collocation points $\left(\mathbf{x}^{\mathrm{i}} \in \Omega\right)$.

Let $\Omega$ be parametrized by a set of $N_{\mathrm{dv}}$ design variables, which can be arranged in a vector as $\mathbf{a}=\left(a_{1}, a_{2}, \ldots, a_{N_{\mathrm{dv}}}\right)^{T}$, i.e. $\Omega=\Omega(\mathbf{a})$. By performing a Taylor's expansion of this parametrization around a given reference domain $\Omega\left(\mathbf{a}^{0}\right)$, it is possible to build a linear mapping of each material point $\mathbf{x}$ of the reference domain $\Omega\left(\mathbf{a}^{0}\right)$ to its position $\tilde{\mathbf{x}}$ in a perturbed domain $\tilde{\Omega}$ as:

$$
\tilde{\mathbf{x}}=\mathbf{x}+\left(\frac{\partial \mathbf{x}}{\partial a_{d}}\right)_{\mathbf{a}^{0}}\left(a_{d}-a_{d}^{0}\right)=\mathbf{x}+\delta \mathbf{x}_{d}\left(a_{d}-a_{d}^{0}\right), d=1, \ldots, N_{\mathrm{dv}}
$$

where $\delta \mathbf{x}_{d}$ denotes the so-called design velocity of the design variable $a_{d}$ at $\mathbf{x}$. Each design velocity defines the region deformation imposed by the design variable with respect to which displacement and traction sensitivities are calculated. As described in Section 1, there are several ways to arrive to a first-order Geometric Sensitivity BEM (GS-BEM). In the present paper, we use the Geometric Sensitivity Singular BIE derived by Rus and Gallego [35] ( $\delta$ SBIE), which is based on a linear Taylor's expansion of Eq. (1) with respect to a given design velocity field. For a single design variable $a$ with design velocity $\delta \mathbf{x}$, it can be written as:

$$
\begin{array}{r}
c_{l k}^{\mathrm{i}} \delta u_{k}^{\mathrm{i}}+b_{l k j m}^{\mathrm{i}} \delta x_{j, m}^{\mathrm{i}} u_{k}^{\mathrm{i}}+\int_{\Gamma} t_{l k}^{*} \delta u_{k} \mathrm{~d} \Gamma+\int_{\Gamma}\left(\delta t_{l k}^{*}+t_{l k}^{*} \delta J\right) u_{k} \mathrm{~d} \Gamma= \\
\int_{\Gamma} u_{l k}^{*} \delta t_{k} \mathrm{~d} \Gamma+\int_{\Gamma}\left(\delta u_{l k}^{*}+u_{l k}^{*} \delta J\right) t_{k} \mathrm{~d} \Gamma
\end{array}
$$

where $l, k, j, m=1,2 ; \delta J$ is the variation of a differential length of boundary $(\mathrm{d} \Gamma)$ imposed by the design velocity field, and $b_{l k j m}^{\mathrm{i}}$ is a free-term which is always null except for non-smooth boundary collocation points with a non-zero design velocity field. The presence of derivatives of the design velocity field in Eq. (6) forces to perform the col- 
location at points where design velocities are $C^{1}$-continuous (differentiable) [35]. This issue is treated in Section 2.2. Left hand side integrals of Eq. (6) must be understood as Cauchy Principal Value integrals $(f)$ when $\mathbf{x}^{\mathrm{i}} \in \Gamma$. The free-term $c_{l k}^{\mathrm{i}}$, and first and third integrals of Eq. (6) are similar to those of Eq. (1), except that, instead of displacements and tractions, their sensitivities appear. The remaining terms contain displacements and tractions, which are already known once the zero-order analysis is performed, and hence constitute load terms. Assuming that displacement and traction sensitivities are interpolated in the same way as displacements and tractions:

$$
\delta u_{k}=\phi_{p}^{(e)} \delta u_{k p}^{(e)}, \delta t_{k}=\phi_{p}^{(e)} \delta t_{k p}^{(e)}
$$

and considering the same collocation setup as in the zero-order analysis, the first-order discretized system can be written as:

$$
\mathbf{H} \delta \mathbf{u}+\delta \mathbf{H} \mathbf{u}=\mathbf{G} \delta \mathbf{t}+\delta \mathbf{G} \mathbf{t}
$$

which could also have been obtained by using a more straightforward semi-analytical approach, see e.g. [39]. However, a semi-analytical approach introduces additional difficulties and uncertainties regarding the selection of appropriate finite-difference schemes and step sizes, leading to a methodology requiring some parameter tuning. After applying the boundary conditions, Eq. (8) leads to the following linear system of equations:

$$
\mathbf{A} \delta \mathbf{x}=\mathbf{B} \delta \breve{\mathbf{x}}+\delta \mathbf{G} \mathbf{t}-\delta \mathbf{H} \mathbf{u}=\mathbf{b}^{\delta}
$$

where $\mathbf{A}$ and $\mathbf{B}$ are exactly the same as in Eq. (4), and the components of $\delta \breve{\mathbf{x}}$ are related to the sensitivities of the boundary conditions. Therefore, it is necessary to build as many load vectors $\mathbf{b}^{\delta}$ as design variables, and then the resulting linear systems of equations are solved. Since Eq. (4) must be solved first, the factorization of $\mathbf{A}$ is already available at this stage, and hence solving Eq. (9) is straightforward.

The previous formulation is given for a simple single region problem for the sake of brevity. The generalization for multi-region problems is analogous to the conventional BEM since compatibility and equilibrium conditions for displacement and traction sensitivities along interfaces are similar to those of displacements and tractions. 


\subsection{Parametrization, design velocity field and collocation technique}

Instead of using the coordinates of corner points as design variables, a set of more meaningful design variables are considered: the coordinates of key reference points of walls, and thicknesses. This avoids defining an otherwise large set of equality constraints during optimization. Therefore, a given vector of design variables $\mathbf{a}=$ $\left(a_{1}, a_{2}, \ldots, a_{N_{\mathrm{dv}}}\right)^{T}$ analytically defines a set of $N_{\mathrm{gp}}$ parametrized geometry points $\mathbf{p}^{(q)}=$ $\mathbf{p}^{(q)}(\mathbf{a}), q=1,2, \ldots, N_{\mathrm{gp}}$. At each iteration and for each frequency, a mesh generator (gmsh [40] is used) produces the boundary element mesh using a pre-defined number of elements per wavelength. This guarantees a valid mesh with an adaptive and minimal number of elements for each analysis.

There are many ways to define the design velocity field, see e.g. [41, 42], and this is the key to obtain reliable sensitivities. Design velocity fields are not uniquely defined, but a number of theoretical and practical conditions must be met [42]. Physically speaking, the design velocity field must be at least $C^{0}$ (continuous) throughout the domain, otherwise the design velocity field breaks the domain. In the absence of body forces and domain information in the optimization problem, only boundary design velocities have to be defined. A design element approach [42] is used since it is appropriate for relatively simple designs. To this end, linear line elements are used as design elements for the parametrization of boundaries. Design velocities are analytically obtained at design nodes from parametrized geometry points $\mathbf{p}^{(q)}=\mathbf{p}^{(q)}(\mathbf{a})$. Therefore, two different meshes coexist for a given problem: a boundary element mesh (discretization of boundary $\mathbf{x}, \mathbf{u}, \mathbf{t}, \delta \mathbf{u}$ and $\delta \mathbf{t}$ ), and a design mesh (discretization of boundary $\mathbf{x}, \delta \mathbf{x})$. A given design element $d e$ establishes:

$$
x_{k}=\psi_{q}^{(d e)} x_{k q}^{(d e)}, \delta x_{k}=\psi_{q}^{(d e)} \delta x_{k q}^{(d e)}
$$

where $\psi_{q}^{(d e)}$ are shape functions, $q=1, \ldots, N_{\mathrm{n}}^{(d e)}$, and $N_{\mathrm{n}}^{(d e)}$ is the number of nodes of design element $d e$. Once Eq. (6) is discretized, shape functions and derivatives of shape functions of design elements appear in the integrals together with shape functions of boundary elements.

As mentioned in the previous section, the Geometric Sensitivity Singular BIE shown in Eq. (6) requires that the design velocity at the collocation point must have an even higher degree of smoothness $\left(C^{1}\right)$. The approach followed by Rus and Gallego [35] is to use standard Lagrange boundary elements and non-nodal collocation along bound- 
aries with non-null design velocities. This approach is very versatile as it automatically guarantees differentiability at the collocation point, but it implies the evaluation of much more integrals than strictly required. In the present work, standard Lagrange boundary elements are also used, but non-nodal collocation is used only where it is required: at double nodes of the boundary element mesh producing a singular coefficient matrix $\mathbf{A}$, and also at nodes where the design velocity field defined by the design mesh is not $C^{1}$. Similarly to Rus and Gallego [35], non-nodal collocation points are moved by $20 \%$ from nodal position.

\subsection{Optimization problem statement}

The problem under consideration is the shape optimization of a wave barrier system located within a rectangular design domain $\left[d_{1}^{\min }, d_{1}^{\max }\right] \times\left[d_{2}^{\min }, d_{2}^{\max }\right] \subset \mathbb{R}^{2}$. The design domain is located between a point source located at $\mathbf{x}^{\mathrm{s}}$, and a point receiver located at $\mathbf{x}^{\mathrm{r}}$, both of them at the half-plane free-surface (see Figure 1). The point source is a unit vertical displacement. The half-plane (soil) and the barrier system are homogeneous, isotropic, linear elastic solids with bonded contact conditions. Time harmonic analyses are used to measure the performance of the wave barrier, which is given in terms of the insertion loss at the receiver location defined as:

$$
I L\left(f, \mathbf{x}^{\mathrm{r}}\right)=20 \log _{10}\left(\frac{\sqrt{\left|u_{1}^{\mathrm{wob}}\left(f, \mathbf{x}^{\mathrm{r}}\right)\right|^{2}+\left|u_{2}^{\mathrm{wob}}\left(f, \mathbf{x}^{\mathrm{r}}\right)\right|^{2}}}{\sqrt{\left|u_{1}^{\mathrm{wb}}\left(f, \mathbf{x}^{\mathrm{r}}\right)\right|^{2}+\left|u_{2}^{\mathrm{wb}}\left(f, \mathbf{x}^{\mathrm{r}}\right)\right|^{2}}}\right)
$$

where $u_{k}$ denotes the displacement component, superscript "wob" stands for "without barrier", superscript "wb" stands for "with barrier", and $f$ is the excitation frequency (in $\mathrm{Hz}$ ). The wave barriers studied are fully described in Section 3.

The problem at hand is a non-convex non-linear constrained optimization problem which can be formulated as:

$$
\begin{array}{llr}
\min _{\mathbf{a}} & \chi \\
\text { s.t. } & a_{j}^{\min } \leq a_{j} \leq a_{j}^{\max }, j=1, \ldots, N_{\mathrm{dv}} & \text { (bounds) } \\
& d_{k}^{\min } \leq p_{k}^{(q)} \leq d_{k}^{\max }, k=1,2, q=1, \ldots, N_{\mathrm{gp}} & \text { (design domain) } \\
& g_{m} \leq 0, m=1, \ldots, N_{\mathrm{c}} & \text { (compatibility) } \\
& A \leq A^{\max } & \text { (economy) }
\end{array}
$$




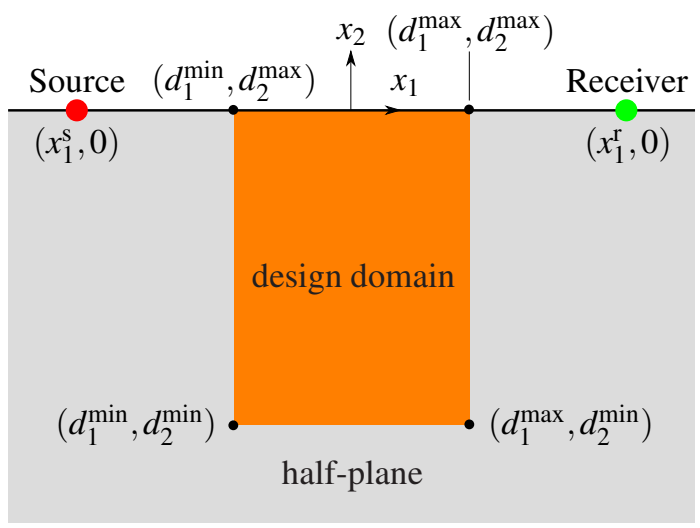

Figure 1: Problem layout

where three different types of objective functions are considered:

$$
\begin{gathered}
\chi=\chi^{\mathrm{s}}=-I L\left(f^{\mathrm{opt}}, \mathbf{x}^{\mathrm{r}}\right) \\
\chi=\chi^{\mathrm{avg}}=-\sum_{i=1}^{\mathrm{N}_{\mathrm{f}}} w_{i} \cdot I L\left(f_{i}^{\mathrm{opt}}, \mathbf{x}^{\mathrm{r}}\right)=-\overline{I L}\left(\left\{f_{1}^{\mathrm{opt}}, \ldots, f_{N_{\mathrm{f}}}^{\mathrm{opt}}\right\}, \mathbf{x}^{\mathrm{r}}\right) \\
\chi=\chi^{\mathrm{mm}}=\max _{i=1, \ldots, N_{\mathrm{f}}}-I L\left(f_{i}^{\mathrm{opt}}, \mathbf{x}^{\mathrm{r}}\right)=-\breve{L}\left(\left\{f_{1}^{\mathrm{opt}}, \ldots, f_{N_{\mathrm{f}}}^{\mathrm{opt}}\right\}, \mathbf{x}^{\mathrm{r}}\right)
\end{gathered}
$$

Four types of constraints are defined: Eq. (12b) establishes the bounds of design variables $a_{j}$, Eq. (12c) restricts the parametrized geometry points within the design domain, Eq. (12d) establishes the geometric compatibility constraints in order to avoid geometric degeneration (see e.g. Eq. (19)), and finally Eq. (12e) imposes an economic constraint by limiting the cross section area of the barrier (amount of material). The first type of objective function defined by Eq. (13) is the insertion loss at a receiver point $\mathbf{x}^{\mathrm{r}}$ for a given frequency $f^{\text {opt }}$, where a change in sign is used in order to formulate a minimization problem. This objective function focuses on obtaining an optimized wave barrier for a fixed single-frequency source. The second type of objective function defined by Eq. (14) is a weighted average insertion loss for a given set of $N_{\mathrm{f}}$ frequencies, each of one weighted by $w_{i}\left(\sum_{i=1}^{N_{\mathrm{f}}} w_{i}=1\right)$, which aims to obtain an optimized wave barrier for a broadband source. The third type of objective function defined by Eq. (15) chooses the worst insertion loss from insertion losses evaluated at a given set of frequencies. It aims at obtaining an optimized wave barrier appropriate for a harmonic source whose frequency can vary within a given frequency range.

The optimization problem is solved using the Optimization Toolbox ${ }^{\mathrm{TM}}$ from the nu- 
merical computing software MATLAB ${ }^{\circledR}$ [43]. For the objective functions defined by Eqs. (13) and (14), the function fmincon with the sqp (Sequential Quadratic Programming) algorithm is used. For the objective function Eq. (15), the function fminimax is used. In this problem many local minima may appear, and a simple multi-start procedure [44] is used to overcome this issue. It is, however, not possible to guarantee that the best local minimum found with this procedure is the global minimum. The procedure consists of studying new randomly generated feasible starting points until the difference between the number of expected local minima (Proposition 2 in [44]) and the number of different local minima found is below a given tolerance ( 0.5 is chosen), or until the number of starting points studied is greater than a certain maximum (50 is chosen). The optimization of a given initial point is halted when it is converging to an already studied basin of attraction. These stopping conditions guarantee global optimization termination with most of the minima found, and a reasonable computational cost. As discussed in [44], it is very interesting to note that, based on Bayesian analysis, the number of starting points to be studied depends on previously found local minima and not on the number of design variables of the problem. The objective function and its gradient are evaluated by means of an in-house BEM and GS-BEM code developed in Fortran following the methodology described in previous sections.

\section{Results and discussion}

In this section, two wave barrier topologies are studied: a single wall barrier, and a double wall barrier. Figure 2 shows the general setting and the wave barrier topologies together with the corresponding design variables $a_{j}$.

The source and receiver are 15 meter apart $\left(\mathbf{x}^{\mathrm{s}}=(-7.5,0), \mathbf{x}^{\mathrm{r}}=(7.5,0)\right)$, and the design domain is 5 meter wide and 8 meter deep $\left(d_{1}^{\min }=-2.5, d_{1}^{\max }=2.5, d_{2}^{\min }=-8\right.$, $d_{2}^{\max }=0$ ). The soil region $\Omega_{\mathrm{s}}$ has a shear modulus $\mu_{\mathrm{s}}=80 \mathrm{MPa}$, Poisson's ratio $v_{\mathrm{s}}=1 / 3$ and density $\rho_{\mathrm{s}}=2000 \mathrm{~kg} \cdot \mathrm{m}^{-3}$. Regions $\Omega_{\mathrm{b}}, \Omega_{\mathrm{b} 1}$ and $\Omega_{\mathrm{b} 2}$ represent elastic regions of the barrier with shear modulus $\mu_{\mathrm{b}}=605 \mathrm{MPa}$, Poisson's ratio $v_{\mathrm{b}}=1 / 4$ and density $\rho_{\mathrm{b}}=2000 \mathrm{~kg} \cdot \mathrm{m}^{-3}$. A conventional hysteretic damping ratio of $5 \%$ is considered for all regions, i.e. $\xi_{\mathrm{s}}=\xi_{\mathrm{b}}=0.05$. Any value between $0 \%$ and $7 \%$ has a limited influence in this type of problems [18].

The range of frequencies of interest is $f \in[20,80] \mathrm{Hz}$, and an evenly spaced set of eleven frequencies $\mathbf{f}^{\text {opt }}=\{20,26,32, \ldots, 80\} \mathrm{Hz}$ is considered for all the objective 


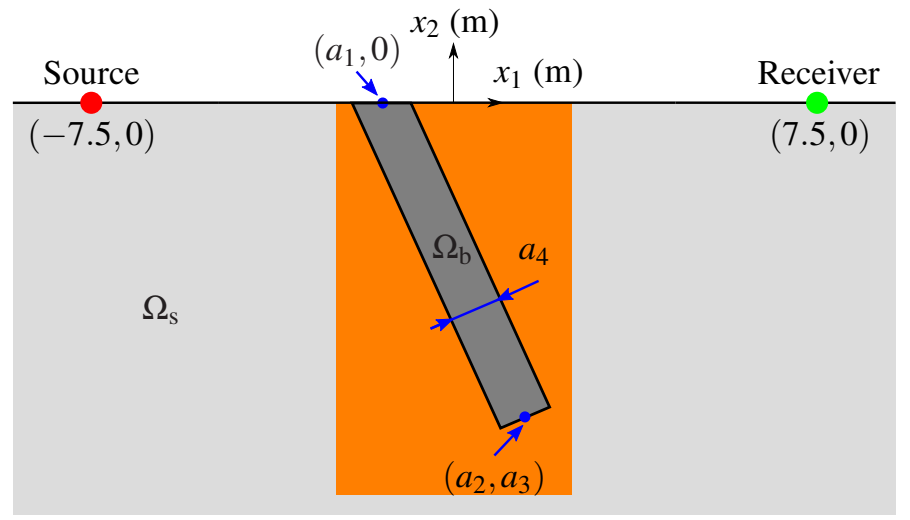

(a) Single wall barrier

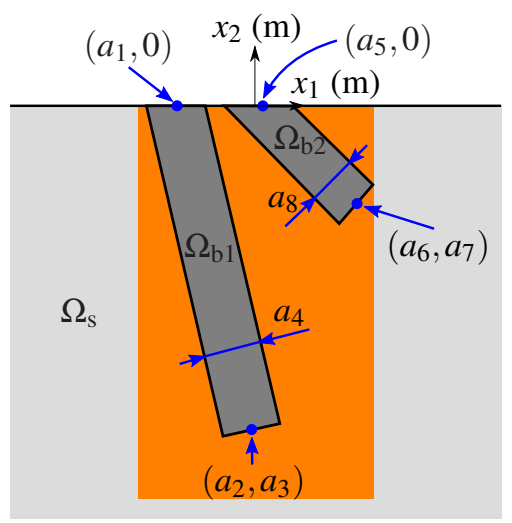

(b) Double wall barrier

Figure 2: Studied wave barrier topologies: (a) single wall barrier, and (b) double wall barrier; both located inside a design domain of $5 \mathrm{~m} \times 8 \mathrm{~m}$ (in orange). 
functions. For the objective function $\chi^{\mathrm{s}}$ defined by Eq. (13), one optimized design per source frequency $f^{\text {opt }}$ in the set is obtained. For objective functions $\chi^{\text {avg }}$ and $\chi^{\mathrm{mm}}$ defined respectively by Eqs. (14) and (15), the frequencies in the set are the sampling points of the otherwise continuous insertion loss spectra, and thus one optimized barrier per objective function is obtained. This relatively coarse frequency sampling provides a good approximation of the continuous spectra while keeping a reasonable computational cost. For objective function $\chi^{\text {avg }}$, a uniform weighting is assumed by default. In the upcoming figures, the insertion loss spectrum for each optimized barrier is calculated for a frequency range $f \in(0,100]$ with steps of $2 \mathrm{~Hz}$.

Ahmad and Al-Hussaini [18] explain the physical phenomena related to vertical stiff wall barriers in two-dimensional problems. In the present case, the shear wave velocities are $c_{\mathrm{Ss}}=200 \mathrm{~m} \cdot \mathrm{s}^{-1}$ and $c_{\mathrm{Sb}}=550 \mathrm{~m} \cdot \mathrm{s}^{-1}$ for the soil and barrier, respectively. They suggested that the ratio of $c_{\mathrm{Sb}} / c_{\mathrm{Ss}}$ should be greater than 2.5 in order to obtain an effective single vertical wall, which is here 2.75 . The Rayleigh wave velocity in the soil is $c_{\mathrm{Rs}}=186.4 \mathrm{~m} \cdot \mathrm{s}^{-1}$. Since the range of frequencies of interest is $f \in[20,80] \mathrm{Hz}$, Rayleigh wavelengths $\lambda_{\mathrm{Rs}}$ vary from $2.33 \mathrm{~m}$ to $9.32 \mathrm{~m}$. Therefore, for the lowest frequencies, the design domain limits the barrier depth up to approximately one Rayleigh wavelength. This will force the optimization to search for solutions that increases the insertion loss without going deeper. For higher frequencies, there is a compromise between depth and width. Ahmad and Al-Hussaini found that an optimal depth to width ratio between 1.2 and 2.8 is obtained when the dimensionless area is $A / \lambda_{\mathrm{Rs}}^{2}>0.2$. They showed that depth and width are both generally relevant. This contrasts with open trenches, where width is almost irrelevant when depth is greater than $0.6 \lambda_{\text {Rs. }}$. Their conclusions give useful design criteria for single vertical wall barriers, and serve as a starting point for the inclined single and double wall studied here.

\subsection{Validation}

In this section, results obtained from different numerical models are compared for the purpose of validation. First, insertion loss results obtained from the present BEM model are compared against results obtained from a FEM-PML model [12]. Secondly, sensitivity results obtained from the present Geometric Sensitivity BEM model are compared against those obtained using Global Finite Differences (GFD) with a BEM model. 


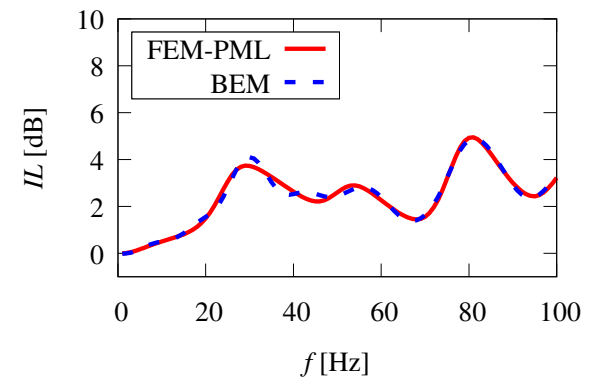

(a) Validation results (single wall barrier)

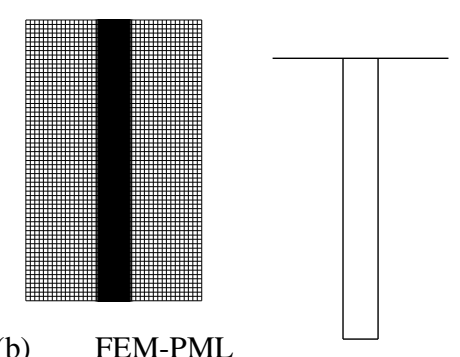

(c) BEM model

Figure 3: Insertion loss $I L(f,(7.5,0) \mathrm{m})$ validation and the corresponding models (only the design domain is shown)

Figure 3 shows insertion loss results of a single wall barrier with $a_{1}=a_{2}=0 \mathrm{~m}$, $a_{3}=-8 \mathrm{~m}$ and $a_{4}=1 \mathrm{~m}$ (see Figure 2). In the near field, a mesh of at least eight linear elements per wavelength is considered in the FEM-PML model in the near field. In the case of the BEM model, at least six quadratic elements per wavelength are considered in the near field, while a progressive change of element size down to three elements per wavelength is used for the far field, and the free-surface is truncated at 50 meters. At the minimum frequency of interest $(20 \mathrm{~Hz})$ the Rayleigh wavelength in the soil is $\lambda_{\mathrm{Rs}}=9.32 \mathrm{~m}$, which means that mesh truncation is produced at $>5 \lambda_{\mathrm{Rs}}$ from the barrier. The source has a width of 0.2 meters, and a unitary vertical displacement is applied. It can be observed that results obtained from both models satisfactorily agree with each other. Therefore, the same number of elements per wavelength, truncation of the freesurface and source width are used for all the following BEM models, unless otherwise stated.

In order to validate the calculation of sensitivities, comparisons between Global Finite Differences using the BEM model and sensitivities obtained from the Geometric Sensitivity BEM have been made for all topologies. For the sake of brevity, only a comparison of the single wall barrier is presented here: a single vertical wall barrier $\left(a_{1}=a_{2}=0 \mathrm{~m}, a_{3}=-8 \mathrm{~m}\right.$ and $\left.a_{4}=1 \mathrm{~m}\right)$, and a single inclined wall barrier $\left(a_{1}=-1.5 \mathrm{~m}, a_{2}=1.5 \mathrm{~m}, a_{3}=-7.5 \mathrm{~m}\right.$ and $\left.a_{4}=1 \mathrm{~m}\right)$; at an excitation frequency of 25 Hz. GFD are calculated using a simple forward finite difference method. Table 1 shows a comparison of sensitivities obtained from GFD with different step sizes, and those obtained from Geometric Sensitivity BEM. The expected rate of convergence of GFD is observed when compared against Geometric Sensitivity BEM. For both the vertical 


\begin{tabular}{|c|c|c|c|c|c|}
\hline Methodology & $\Delta a_{j}$ & $\frac{\mathrm{d} \chi}{\mathrm{d} a_{1}}$ & $\frac{\mathrm{d} \chi}{\mathrm{d} a_{2}}$ & $\frac{\mathrm{d} \chi}{\mathrm{d} a_{3}}$ & $\frac{\mathrm{d} \chi}{\mathrm{d} a_{4}}$ \\
\hline \multicolumn{6}{|c|}{ Vertical wall $\left(\mathbf{a}=(0,0,-8,0.5)^{T}\right)$ with $\chi=-1.78 \mathrm{~dB}:$} \\
\hline \multirow{6}{*}{ GFD / BEM } & $10^{-1}$ & -0.304962 & -0.020966 & 0.076265 & -2.421967 \\
\hline & $10^{-2}$ & -0.326238 & -0.028191 & 0.051777 & -2.548937 \\
\hline & $10^{-3}$ & -0.328296 & -0.028917 & 0.049330 & -2.562605 \\
\hline & $10^{-4}$ & -0.328497 & -0.028976 & 0.049090 & -2.563981 \\
\hline & $10^{-5}$ & -0.328518 & -0.028983 & 0.049066 & -2.564119 \\
\hline & $10^{-6}$ & -0.328518 & -0.028985 & 0.049064 & -2.564133 \\
\hline GS-BEM & - & -0.328519 & -0.028984 & 0.049063 & -2.564136 \\
\hline \multicolumn{6}{|c|}{ Inclined wall $\left(\mathbf{a}=(-1.5,1.5,-7.5,1)^{T}\right)$ with $\chi=-1.29 \mathrm{~dB}$ : } \\
\hline \multirow{6}{*}{ GFD / BEM } & $10^{-1}$ & -0.395605 & 0.398573 & 0.266008 & -1.751849 \\
\hline & $10^{-2}$ & -0.366735 & 0.400251 & 0.255849 & -1.756983 \\
\hline & $10^{-3}$ & -0.363839 & 0.400423 & 0.254817 & -1.754901 \\
\hline & $10^{-4}$ & -0.363549 & 0.400440 & 0.254713 & -1.754691 \\
\hline & $10^{-5}$ & -0.363520 & 0.400441 & 0.254703 & -1.754671 \\
\hline & $10^{-6}$ & -0.363515 & 0.400443 & 0.254700 & -1.754666 \\
\hline GS-BEM & - & -0.363517 & 0.400442 & 0.254702 & -1.754668 \\
\hline
\end{tabular}

Table 1: Validation of sensitivities for objective function $\chi=-I L(25 \mathrm{~Hz},(7.5,0) \mathrm{m})$

and the inclined wall barriers, the objective function is most sensitive to the thickness design variable $a_{4}$, which basically controls the stiffness of the barrier. Similarly, the objective function is least sensitive to the vertical coordinate of the wall bottom $a_{3}$. This may be explained by the fact that for walls somewhat deeper than one Rayleigh wavelength, $\lambda_{\mathrm{Rs}}=7.456 \mathrm{~m}$ in this case, the insertion loss improvement is small [18].

\subsection{Optimized single wall barriers}

The geometry of a general single wall barrier is controlled by four design variables $\left(N_{\mathrm{dv}}=4\right): a_{1}$ is the horizontal coordinate of the wall top $\left(-2.5 \leq a_{1} \leq 2.5\right), a_{2}$ and $a_{3}$ are respectively the horizontal and vertical coordinates of the wall bottom $(-2.5 \leq$ $\left.a_{2} \leq 2.5,-8 \leq a_{3} \leq-0.1\right)$, and $a_{4}$ is the wall thickness $\left(0.1 \leq a_{4} \leq 5\right)$; see Figure 2a. There are four parametrized geometry points $\left(N_{\mathrm{gp}}=4\right)$, which can be be obtained from:

$$
\begin{aligned}
& \mathbf{p}^{(1)}=\left(a_{1}+\left(a_{4} / 2\right) / \cos \theta, 0\right), \mathbf{p}^{(2)}=\left(a_{2}+\left(a_{4} / 2\right) \cos \theta, a_{3}+\left(a_{4} / 2\right) \sin \theta\right) \\
& \mathbf{p}^{(3)}=\left(a_{2}-\left(a_{4} / 2\right) \cos \theta, a_{3}-\left(a_{4} / 2\right) \sin \theta\right), \mathbf{p}^{(4)}=\left(a_{1}-\left(a_{4} / 2\right) / \cos \theta, 0\right)
\end{aligned}
$$

where $\theta=\arctan \left(\left(a_{1}-a_{2}\right) / a_{3}\right)$. These points correspond to the top right, bottom right, bottom left and top left corner points of the wall, respectively. The bounds established previously for design variables are less strict than those of the points $\mathbf{p}^{(q)}$ (Eq. (12c)), 
in such a way that the design space allows any barrier within the design domain. This is also done for the double wall barrier.

\subsubsection{Detailed example runs of optimization}

In this section, detailed results of the optimization procedure are shown in order to illustrate its main features and performance. Only the objective function related to single-frequency sources (Eq. (13)) is considered, and detailed results for harmonic sources at $20 \mathrm{~Hz}, 50 \mathrm{~Hz}$ and $80 \mathrm{~Hz}$ are described. A maximum area of $A_{\max }=8 \mathrm{~m}^{2}$ is assumed in all cases. The computation times are obtained using a desktop computer with a $3.7 \mathrm{GHz}$ two cores processor.

Figures $4 \mathrm{a}, 4 \mathrm{c}$ and $4 \mathrm{e}$ illustrate respectively for harmonic sources at $20 \mathrm{~Hz}, 50$ $\mathrm{Hz}$ and $80 \mathrm{~Hz}$ the convergence, the expected (Proposition 2 in [44]) and the number of local minima found, and computation time of the global optimization algorithm as the number of initial points considered increases. It is observed that most of the local minima are found in the beginning. Later, the global optimization stops once the difference between the number of expected local minima and the number of local minima found is below 0.5 , as was previously indicated in Section 2.3. The total run time increases considerably with the frequency of the harmonic source considered in the optimization, from about 5 minutes for $f^{\text {opt }}=20 \mathrm{~Hz}$ to 30 minutes for $f^{\text {opt }}=80$ $\mathrm{Hz}$.

Figure $4 \mathrm{~b}, 4 \mathrm{~d}$ and $4 \mathrm{f}$ depict respectively for harmonic sources at $20 \mathrm{~Hz}, 50 \mathrm{~Hz}$ and $80 \mathrm{~Hz}$ the convergence of the optimization from the initial point leading to the best local minimum found in each case. In about the first half of iterations a quick convergence to the minimum is observed followed by a solution refinement in order to achieve the established tolerance $\left(10^{-4}\right)$.

\subsubsection{Optimized reference cases}

The first optimization problem to be considered is the simplified case of a vertical and centered wall obtained from the general case stated above by setting $a_{1}=a_{2}=0$. The aim is studying to what extent a simple one Rayleigh wavelength deep wall is optimal. Also, since these are the simplest single wall barrier designs to install, it is appropriate to compare the performance of more involved designs with such simple ones. Because of that, these are referred as the optimized reference cases in the rest of the paper. Optimization is performed for four different maximum area constraints 

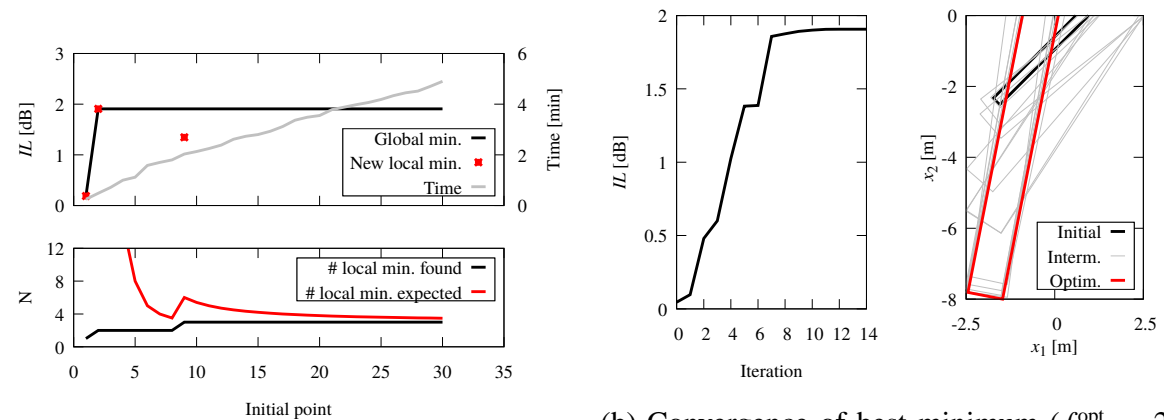

(a) Global convergence $\left(f^{\text {opt }}=20 \mathrm{~Hz}\right)$

(b) Convergence of best minimum ( $f^{\text {opt }}=20$

$\mathrm{Hz})$
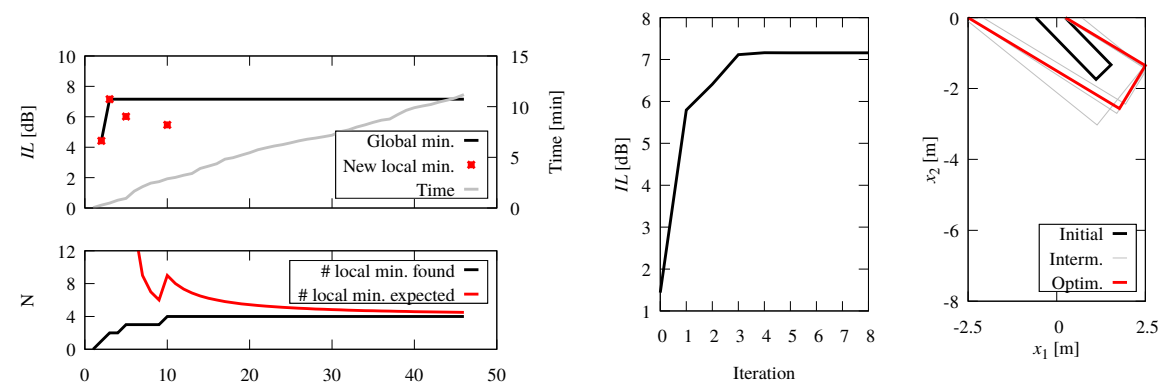

(d) Convergence of best minimum ( $f^{\text {opt }}=50$

(c) Global convergence $\left(f^{\text {opt }}=50 \mathrm{~Hz}\right)$

$\mathrm{Hz})$
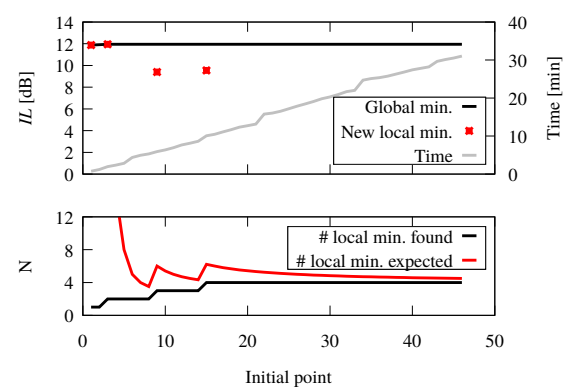

(e) Global convergence $\left(f^{\mathrm{opt}}=80 \mathrm{~Hz}\right)$
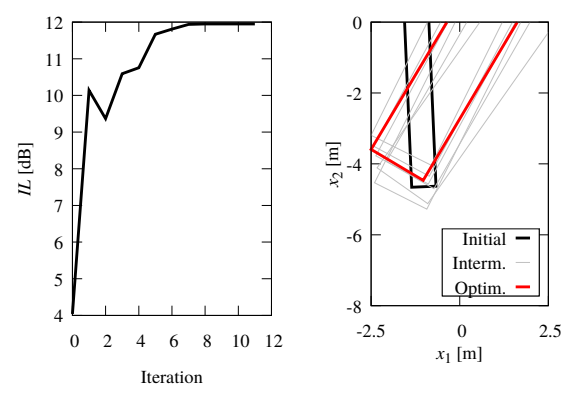

(f) Convergence of best minimum $\left(f^{\text {opt }}=80\right.$ $\mathrm{Hz})$

Figure 4: Detailed example runs for single-frequency sources $\left(A_{\max }=8 \mathrm{~m}^{2}\right)$ 

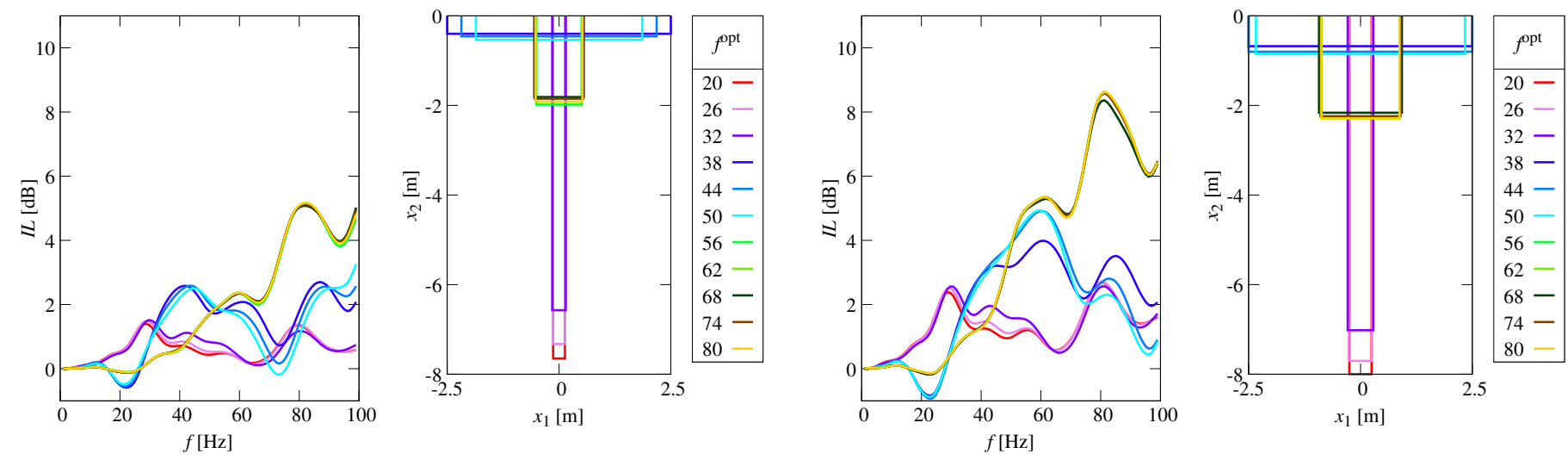

(a) $A_{\max }=2 \mathrm{~m}^{2}$
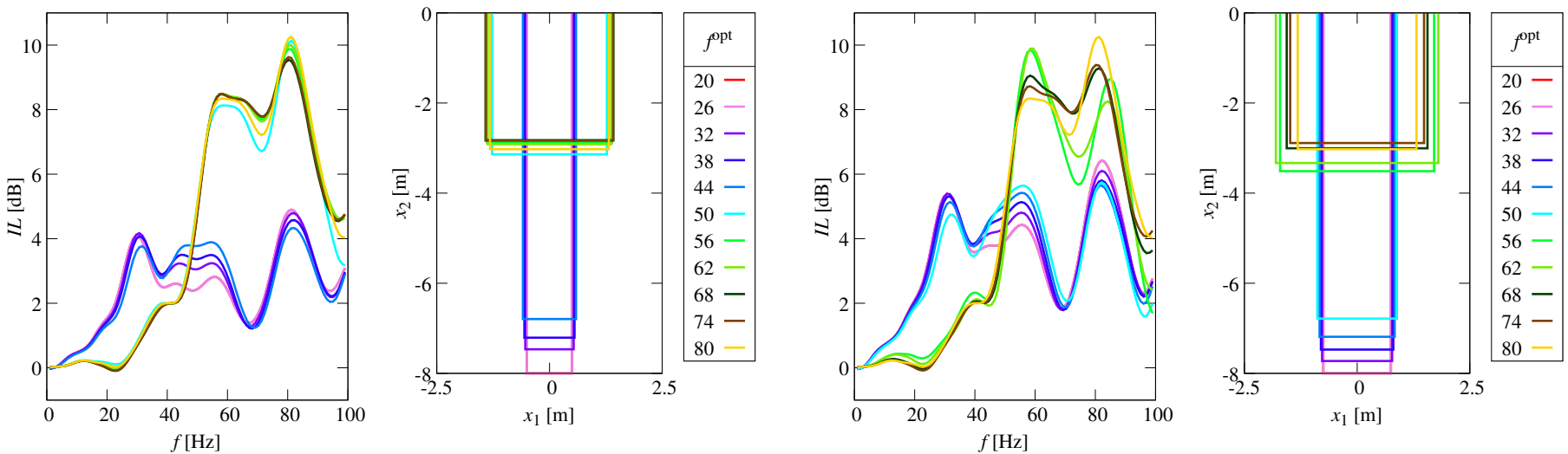

(c) $A_{\max }=8 \mathrm{~m}^{2}$

(d) $A_{\max }=12 \mathrm{~m}^{2}$

Figure 5: Optimized vertical and centered single wall barriers for single-frequency sources

$A_{\max }=\{2,4,8,12\} \mathrm{m}^{2}$, and thus the dimensionless area is within $0.025<A_{\max } / \lambda_{\mathrm{R}}^{2}<$ 2.22 .

For the objective function in Eq. (13) related to single-frequency sources, the obtained global minima are depicted in Figure 5 together with their insertion loss spectra. Three types of optimized designs with different aspect ratios are found: deep vertical walls (optimal for low frequencies), slabs (optimal for mid frequencies in some cases), and thick walls (optimal for high-frequencies). Results show that there is no smooth transition between these types of optimized designs as the target frequency increases. In fact, these are more or less similar within a frequency interval, and only small changes take place in order to achieve optimal performance at each target frequency. The frequencies at which the type of optimal design shifts depend on the amount of material available $\left(A_{\max }\right)$. For $A_{\max }=8$ and $A_{\max }=12 \mathrm{~m}^{2}$, there is a sudden 


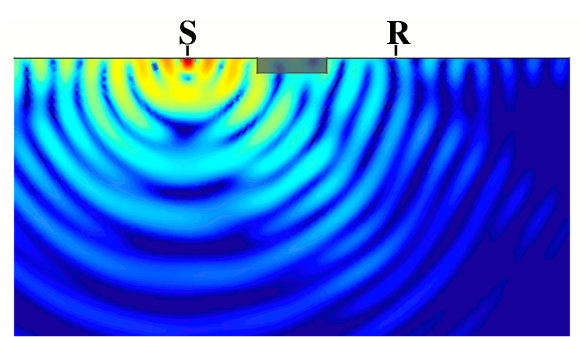

S

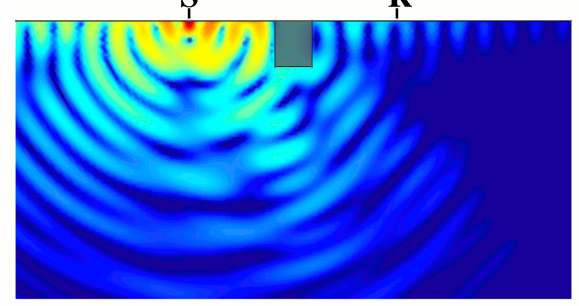

S R

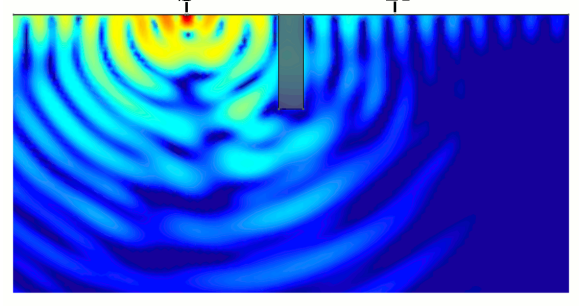

0.01
0.10

$|\operatorname{Re}(\mathbf{u})|[\mathrm{m}]$

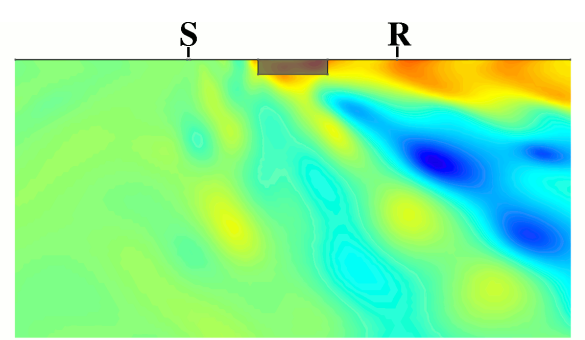

R

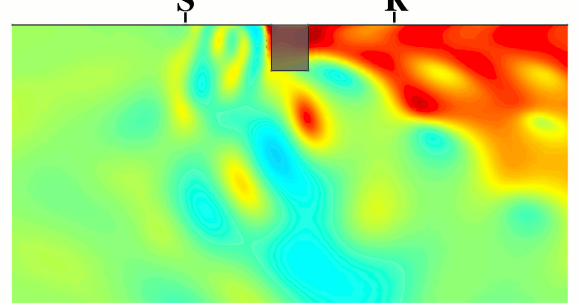

R

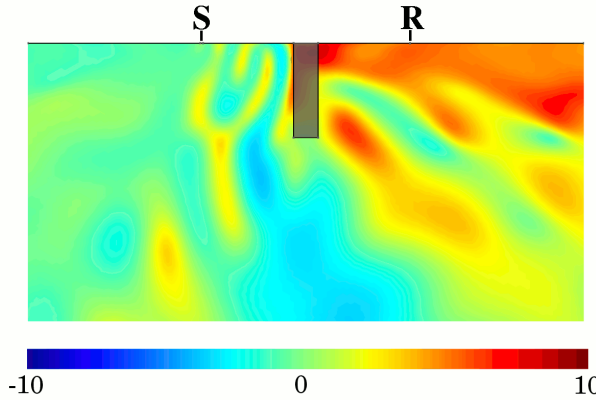

$I L[\mathrm{~dB}]$

Figure 6: Comparison between three approximately similar local minima: slab (top), thick wall (middle), and deep wall (bottom); $\left(f^{\mathrm{opt}}=50 \mathrm{~Hz}, A_{\max }=12 \mathrm{~m}^{2}\right)$

shift from deep walls to a thick walls at approximately $50 \mathrm{~Hz}\left(\lambda_{\mathrm{R}}=3.728 \mathrm{~m}\right)$. For $A_{\max }=2$ and $A_{\max }=4 \mathrm{~m}^{2}$, there is a shift from deep walls to slabs at approximately $30 \mathrm{~Hz}$, and then from slabs to thick walls at approximately $50 \mathrm{~Hz}$. These three types of optimized designs appear in the set of local minima in each case, but one of them becomes the optimal depending on the frequency of the source. This explains the sudden shifts between these designs as the frequency of the harmonic source changes. This behavior is explained further in the next paragraph. It can be concluded that the rule of thumb of limiting the depth of the barrier at one Rayleigh wavelength generally does not lead to an optimal barrier for a given amount of material.

In order to compare the differences between all three kinds of designs, Figure 6 shows color maps of the magnitude of the real part of displacement and insertion losses within a domain of interest $\left(-20 \leq x_{1} \leq 20 \times-20 \leq x_{2} \leq 0 \mathrm{~m}^{2}\right)$ for the three local minima of the case $f^{\text {opt }}=50 \mathrm{~Hz}, A_{\max }=12 \mathrm{~m}^{2}$ : slab design $\left(a_{1}=a_{2}=0 \mathrm{~m}\right.$, 

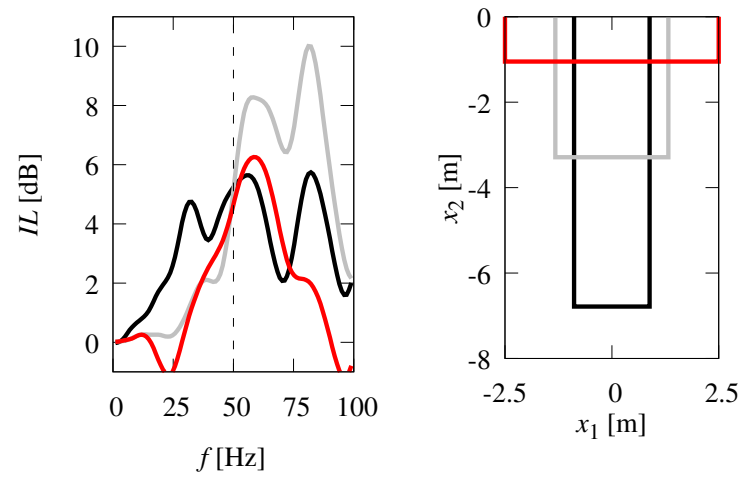

Figure 7: Insertion loss spectra of three approximately similar local minima: slab (red), thick wall (grey), and deep wall (black); $\left(f^{\text {opt }}=50 \mathrm{~Hz}, A_{\max }=12 \mathrm{~m}^{2}\right)$

$\left.a_{3}=-1.05 \mathrm{~m}, a_{4}=5 \mathrm{~m}, I L=4.7 \mathrm{~dB}\right)$, thick wall design $\left(a_{1}=a_{2}=0 \mathrm{~m}, a_{3}=-3.288\right.$

$\left.\mathrm{m}, a_{4}=2.642 \mathrm{~m}, I L=5.1 \mathrm{~dB}\right)$, and deep wall design $\left(a_{1}=a_{2}=0 \mathrm{~m}, a_{3}=-6.785\right.$

$\mathrm{m}, a_{4}=1.769 \mathrm{~m}, I L=5.2 \mathrm{~dB}$ ). This case is interesting because these three very different designs have insertion losses around $5 \mathrm{~dB}$ at the receiver location and target frequency $f^{\text {opt }}$ (see Fig. 7). The Rayleigh wavelength in the soil is $\lambda_{\mathrm{Rs}}=3.7 \mathrm{~m}$ at $50 \mathrm{~Hz}$. The width of the slab covers the full design domain, which is $1.35 \lambda_{\mathrm{Rs}}$, but the depth $0.28 \lambda_{\mathrm{Rs}}$ is smaller than allowed by the available area $\left(A=5.25 \mathrm{~m}^{2}<A_{\max }\right)$. The depth of the deep wall is $1.8 \lambda_{\mathrm{Rs}}$, and $0.88 \lambda_{\mathrm{Rs}}$ in the case of the thick wall. Therefore, the maximum dimension of the barrier is approximately equal or greater than one Rayleigh wavelength. In Figure 6, it can be seen that the slab diverts incoming waves, which are sent beneath the receiver into the halfspace. The overall effect is a significant insertion loss near the surface along the receiver side. The thick wall partially converts incoming Rayleigh waves into body waves by reflecting them on the wall and also sending body waves through the bottom of the wall into the halfspace. The deep wall makes both types of conversion, but reflection is predominant in this case. Despite having similar insertion losses at the receiver point, the thick wall design has better insertion losses along all the receiver side whereas the slab design has a more localized effect. Figure 7 also shows that the slab design is effective only in a narrower range of frequencies than thick or deep walls.

Figure 8 shows the difference between optimized designs and typical designs with depth of one Rayleigh wavelength for a given target frequency. It shows that optimization leads to a better performance within the range of target frequencies studied. Improvements between 1 and $2 \mathrm{~dB}$ are achievable, which lead to significant improve- 


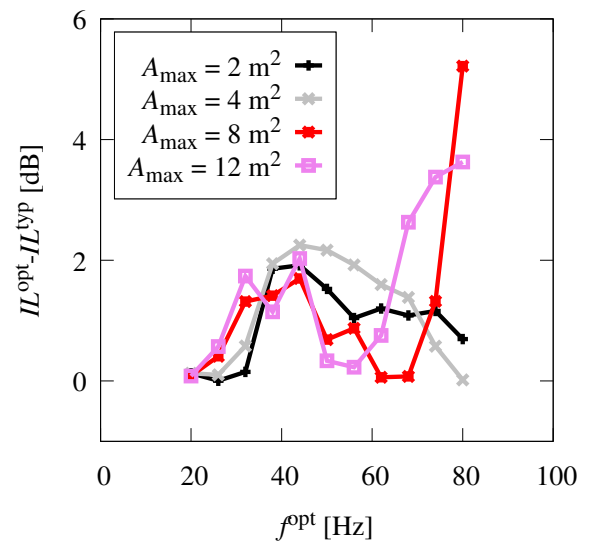

Figure 8: Insertion loss improvement of optimized design (see Figure 5) with respect to typical designs $\left(a_{3}=-\lambda_{\mathrm{R}}\left(f^{\mathrm{opt}}\right), a_{4}=A_{\max } / \lambda_{\mathrm{R}}\left(f^{\mathrm{opt}}\right)\right)$ for each target frequency $f^{\text {opt }}$

ments of $11 \%$ to $21 \%$ in terms of Amplitude Reduction ratios.

For the objective function in Eq. (14) with uniform weighting $\left(w_{i}=1 / N_{\mathrm{f}}\right)$ related to optimization for broadband sources, the obtained global minima are depicted in Figure 9 together with their insertion loss spectra. Since the objective function is an average of insertion losses within a given frequency range, optimization improves insertion losses where they can be easily increased, i.e. at high frequencies. In fact, optimized designs for this objective function are very similar to those of high frequency harmonic sources. There is a clear improvement as the maximum area is increased, except for the largest area considered $\left(A_{\max }=12 \mathrm{~m}^{2}\right)$. The optimal solution in this case does not make use of all available area, which was also the case for some of the previous high frequency harmonic sources. The reason behind this is that, compared to the optimized design, insertion losses in the high frequency range decrease considerably, and in the low frequency range increase slightly when the depth of the barrier is increased to reach $A=A_{\max }$, see Figure 10. Conversely, when the width of the barrier is increased to reach $A=A_{\max }$, insertion losses in the high frequency range increase and in the low frequency range decrease.

For the objective function in Eq. (15) related to optimization for harmonic sources within a frequency range, the obtained global minima are depicted in Figure 11 together with their insertion loss spectra. There are no practical design rules for this objective function, and thus optimization is quite necessary in this case. However, since the lowest insertion losses are usually but not always located at the lowest fre- 

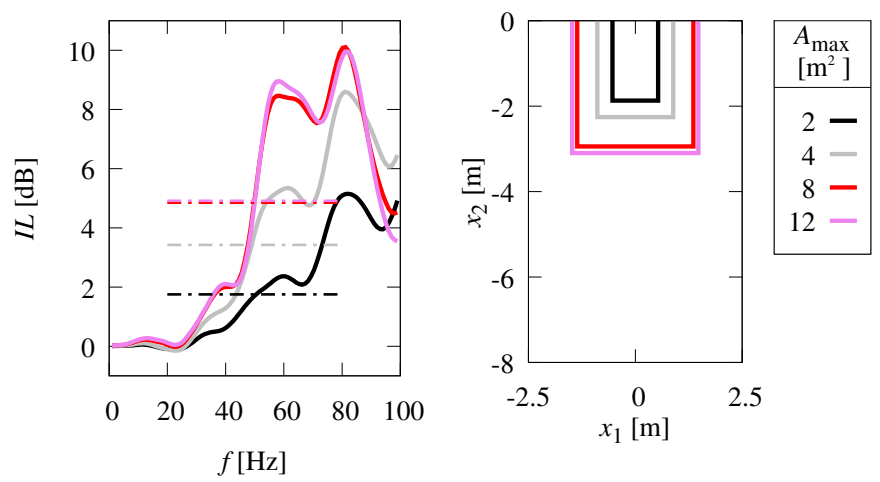

Figure 9: Optimized vertical and centered single wall barriers for broadband sources $\left(f^{\mathrm{opt}}=[20,80] \mathrm{Hz}\right)$
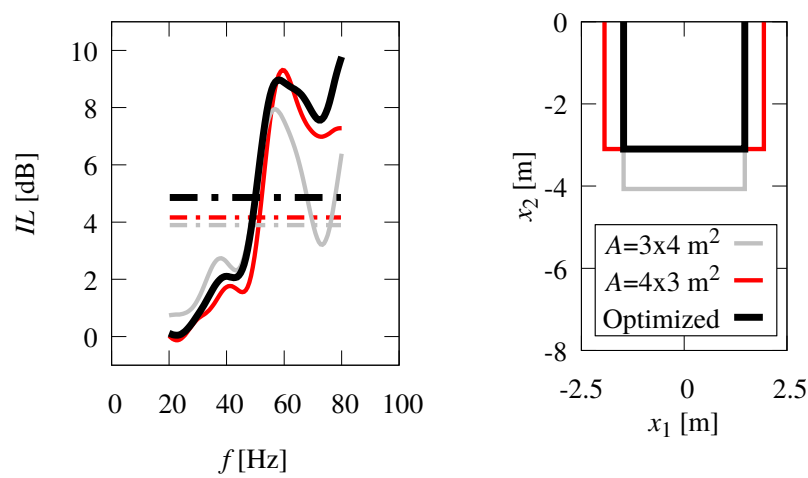

Figure 10: Effect of increasing width or depth for the optimized vertical and centered single wall barrier for broadband sources $\left(f^{\mathrm{opt}}=[20,80] \mathrm{Hz}, A_{\max }=12 \mathrm{~m}^{2}\right)$
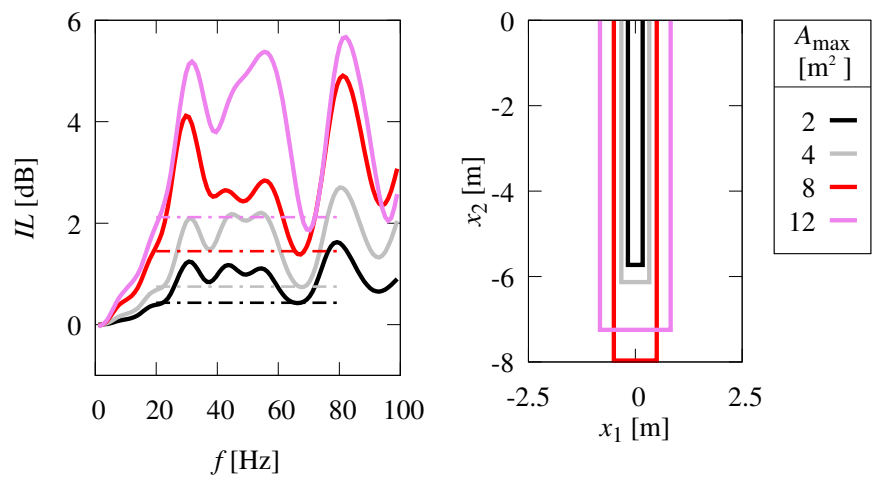

Figure 11: Optimized vertical and centered single wall barriers for harmonic sources within a frequency range $\left(f^{\text {opt }}=[20,80] \mathrm{Hz}\right)$ 
quency, good starting points are those barriers designed for single-frequency sources at the lowest frequency. The results show that the optimized designs are indeed similar to those obtained for single-frequency sources at the lowest frequency. Since the objective function is uniformly sampled from 20 to $80 \mathrm{~Hz}$ in steps of $6 \mathrm{~Hz}$, the actual minimum insertion loss in this frequency range may be lower, as can be seen in Figure 11. As expected, the optimized designs achieve better minimal insertion losses as $A_{\max }$ increases.

\subsubsection{Optimized single wall barriers}

In this section, single wall barriers are optimized considering all four design variables. Compared to the previous case, the horizontal positions of the top and bottom parts of the wall are now included in the optimization, which allows changing the horizontal position and angle of the wall.

The optimized designs for single-frequency sources of 20,26, 44, 50, 74 and 80 $\mathrm{Hz}$ are shown in Figure 12. For low frequency sources, the optimized walls have a small inclination and reach the maximum depth. For mid frequency sources, the optimized walls are highly inclined towards the receiver and cover the entire width of the design domain. For sources of 38 (not shown) and $44 \mathrm{~Hz}$ and the highest values of $A_{\max }$, slightly inclined deep walls are obtained. Depths reached for the former cases are around 2 meters, which is much less that one wavelength. In this sense, these designs can be seen as an evolution of slab designs obtained in several optimized reference cases. A more sophisticated version of this type of design was also obtained by Van hoorickx et al. [12] via topology optimization. For high frequency sources, the optimized walls are highly inclined towards the source and cover almost all the entire width of the design domain. In some cases, nearly square blocks also appear as optimized designs. In the former cases, the depth reached by walls is approximately 4 meters, more than one Rayleigh wavelength. This type of design also appears in a more complicated fashion in [12]. Most of the optimized designs for the same frequency and different values of $A_{\max }$ have very similar inclination and position, showing that these are key factors for tuning the design for the single-frequency source.

The improvement of the obtained global minima with respect to the optimized reference cases is depicted in Figure 13. It is observed that the improvement is small for low frequencies, and becomes more important as the frequency increases, although the increase is not uniform. For frequencies higher than $40 \mathrm{~Hz}$, an improvement between 

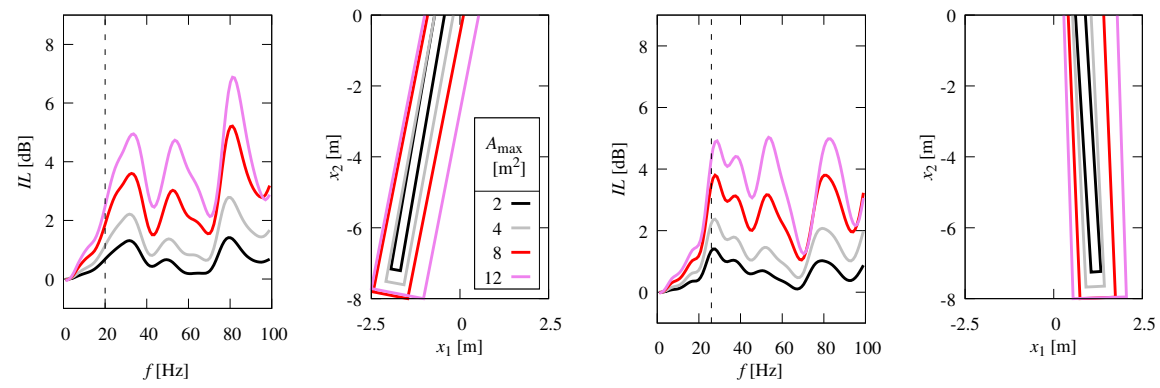

(a) $f^{\text {opt }}=20 \mathrm{~Hz}$
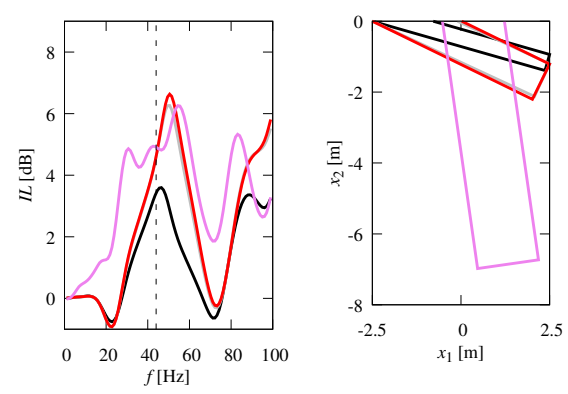

(b) $f^{\text {opt }}=26 \mathrm{~Hz}$

(c) $f^{\text {opt }}=44 \mathrm{~Hz}$
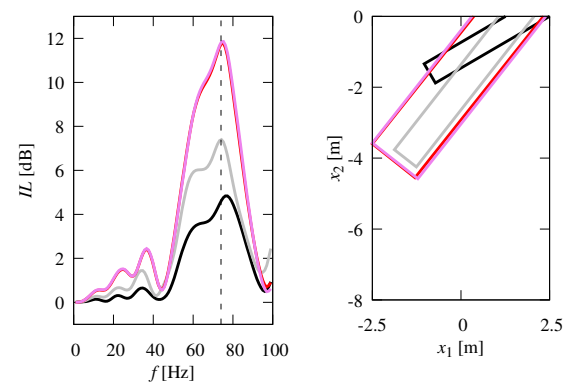

(e) $f^{\text {opt }}=74 \mathrm{~Hz}$
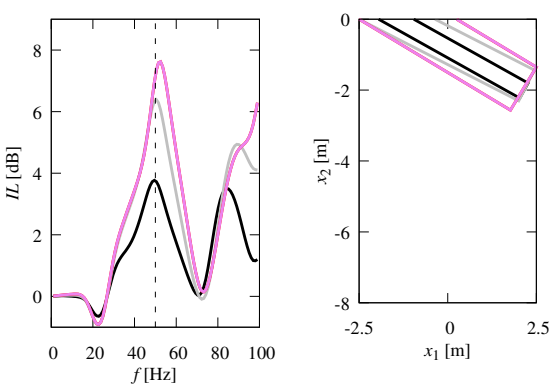

(d) $f^{\text {opt }}=50 \mathrm{~Hz}$
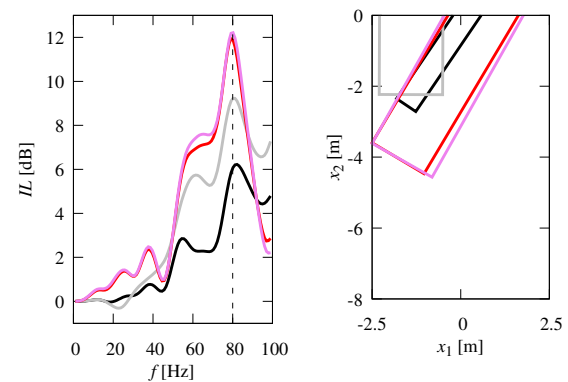

(f) $f^{\text {opt }}=80 \mathrm{~Hz}$

Figure 12: Optimized single wall barriers for single-frequency sources 


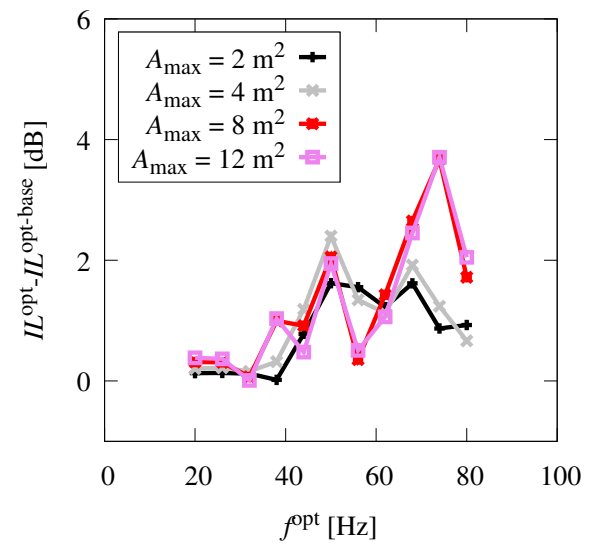

Figure 13: Insertion loss improvement of optimized single wall barriers with respect to optimized reference cases for each target frequency $f^{\text {opt }}$

roughly 1 and $4 \mathrm{~dB}$ can be achieved.

Figure 14 shows the insertion loss color maps for the optimized reference cases and the optimized single wall barriers for single-frequency sources of 50 and $74 \mathrm{~Hz}$, with $A_{\max }=8 \mathrm{~m}^{2}$, and within a given domain of interest $\left(-20 \leq x_{1} \leq 20 \times-20 \leq x_{2} \leq 0\right.$ $\mathrm{m}^{2}$ ). For both single-frequency sources, the optimized single wall barriers relocate insertion loss maxima occurring along the receiver side such that one maximum is placed at the receiver point. Although the optimization is performed for a single receiver point, insertion losses are also significantly improved all along the receiver side. The optimized single wall barrier inclined towards the receiver side $\left(f^{\text {opt }}=50 \mathrm{~Hz}\right)$ reflects incoming waves and also acts as a waveguide redirecting waves away from the surface. The insertion loss map of the optimized single wall barrier inclined towards the source side $\left(f^{\text {opt }}=74 \mathrm{~Hz}\right)$ is essentially similar to the one of a deep wall, see e.g. Figure 6, with the additional inclination improving the insertion losses behind the wall.

For objective functions in Eqs. (14) and (15) related to optimization for broadband sources and for harmonic sources within a frequency range respectively, the obtained global minima are depicted in Figs. 15 and 16 together with their insertion loss spectra. Improvements with respect to the respective optimized reference cases (Figs. 9 and 11) are less than $0.5 \mathrm{~dB}$ for both objective functions, which is a relatively small gain. Resulting designs for a non-uniform weighting are also shown in Fig. 15b. Weight vector $\mathbf{w}=\{1 / 24,2 / 24,4 / 24,6 / 24,4 / 24,2 / 24,1 / 24, \ldots, 1 / 24\}$ is used respectively for frequencies $\mathbf{f}^{\mathrm{opt}}=\{20,26,32,38,44,50,56, \ldots, 80\} \mathrm{Hz}$. It should be noted that 


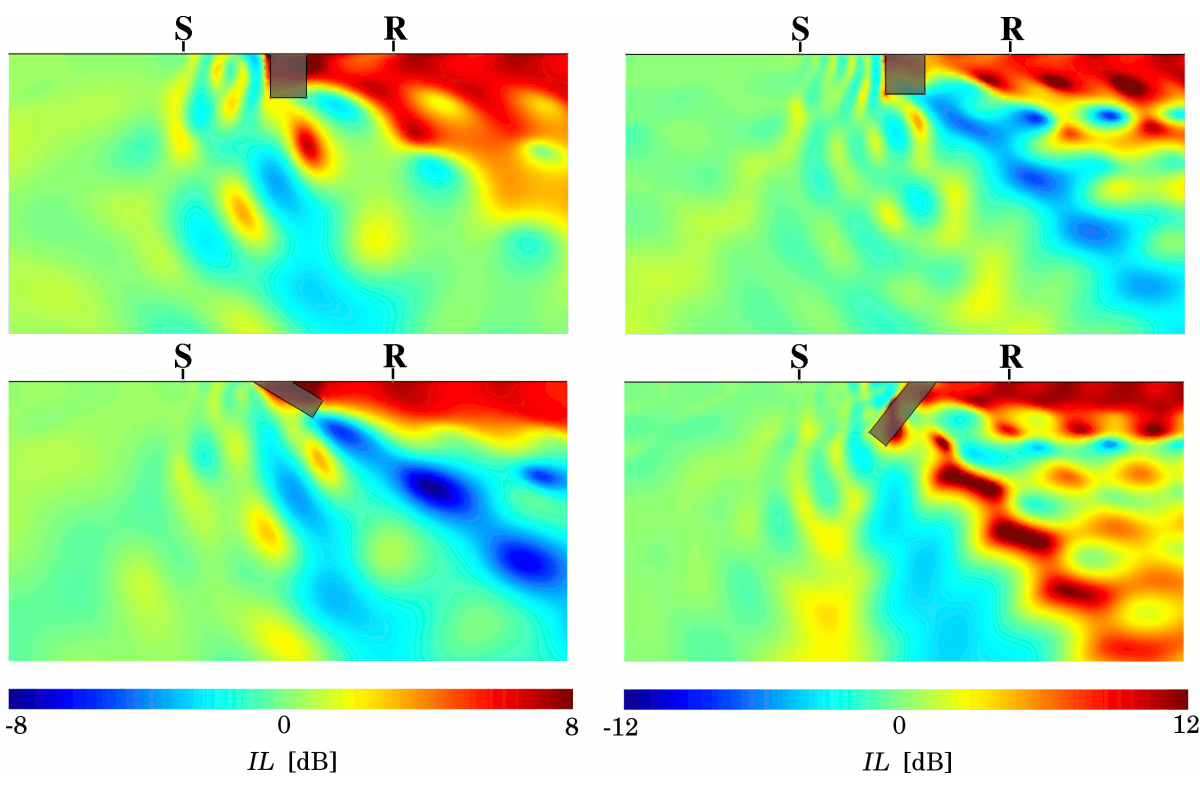

Figure 14: Comparison between $I L$ color maps of optimized reference cases (top) and optimized single wall barriers (bottom) for $f^{\text {opt }}=50 \mathrm{~Hz}$ (left) and $f^{\text {opt }}=74 \mathrm{~Hz}$ (right) when $A_{\max }=8 \mathrm{~m}^{2}$

$75 \%$ of the total weight is concentrated between 26 and $50 \mathrm{~Hz}$. Optimized designs in this case are very similar to those of single-frequency sources for 44 and $50 \mathrm{~Hz}$ (Figs. $12 \mathrm{c}$ and $12 \mathrm{~d}$ ). As was observed before, the designs are very similar to those obtained for single-frequency sources at the highest frequencies of the most relevant frequency window $([26,50] \mathrm{Hz})$.

\subsubsection{Robustness of optimized designs with regard to source and receiver locations}

Objective functions are built to serve a specific purpose, and, in this work, a single point source and a single point receiver are considered. However, in the light of the obtained results, it is relevant to assess the performance of these designs for other source and receiver locations, and thus check their robustness with regard to these factors.

Previously shown $I L$ color maps, e.g. Fig. 14, illustrate that a good vibration reduction is achieved beyond the receiver location. However, as a consequence of the defined objective function, a stronger reduction is achieved near the receiver position. For some representative cases of previously shown optimized designs for $x_{1}^{\mathrm{x}}=-7.5$ m, Fig. 17 depicts point-wise insertion losses along a receiver zone and cumulative 


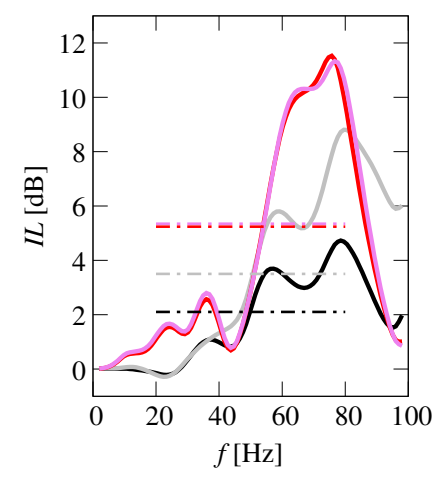

(a) Uniform weighting
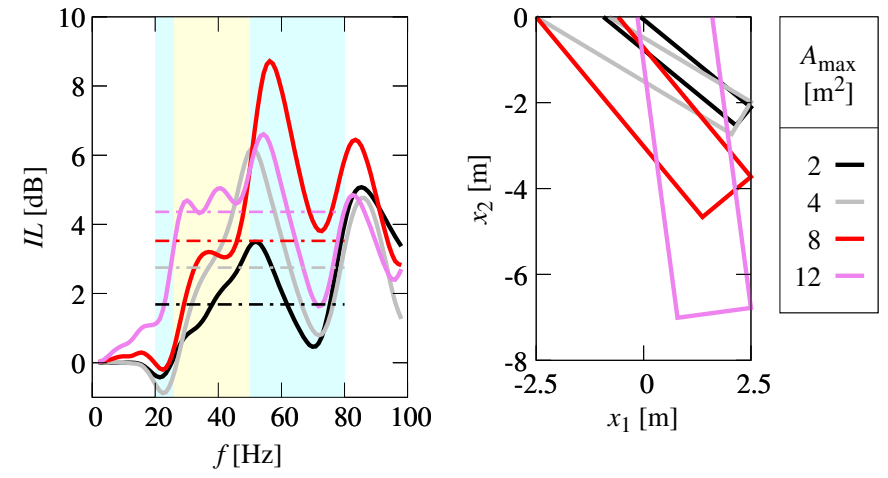

(b) Non-uniform weighting (75\% of weight in $f^{\text {opt }}=[26,50] \mathrm{Hz}$ )

Figure 15: Optimized single wall barriers for broadband sources $\left(f^{\text {opt }}=[20,80] \mathrm{Hz}\right)$
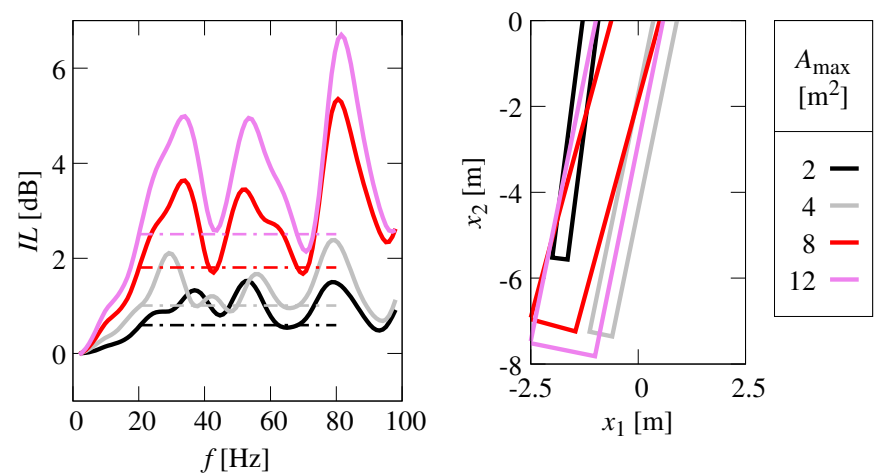

Figure 16: Optimized single wall barriers for harmonic sources within a frequency range $\left(f^{\mathrm{opt}}=[20,80]\right.$ $\mathrm{Hz})$ 

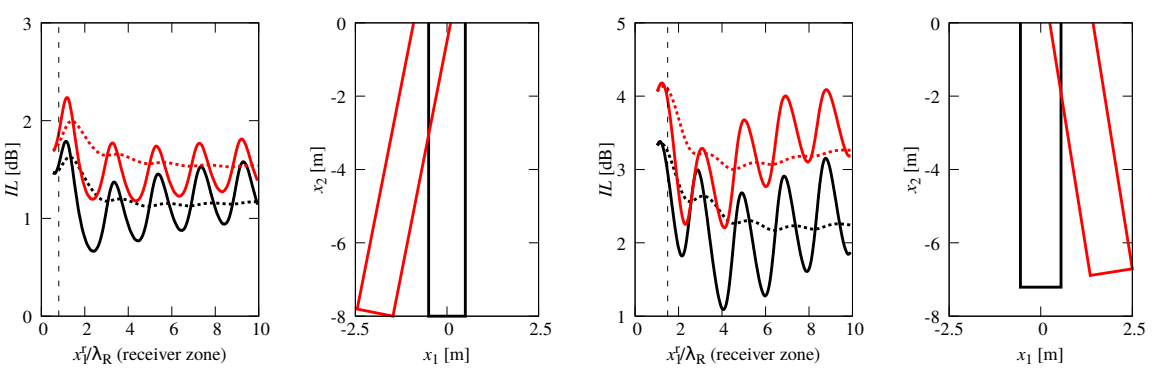

(a) $f^{\text {opt }}=20 \mathrm{~Hz}$

(b) $f^{\text {opt }}=38 \mathrm{~Hz}$
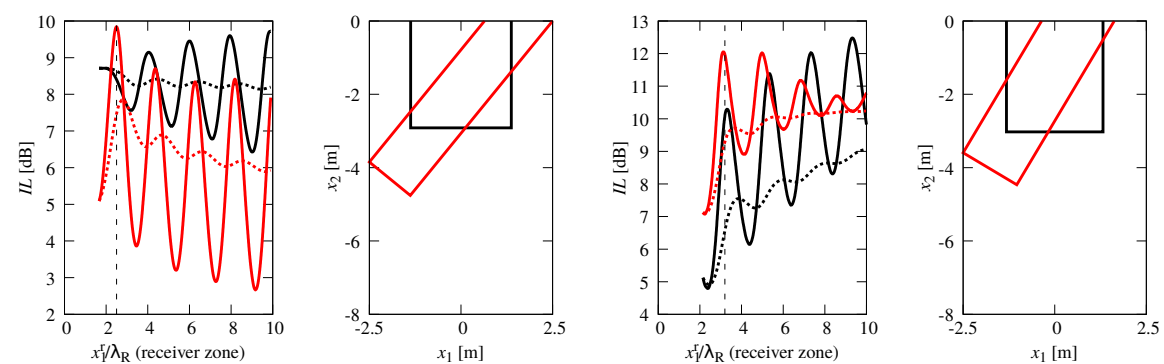

(c) $f^{\text {opt }}=62 \mathrm{~Hz}$

(d) $f^{\text {opt }}=80 \mathrm{~Hz}$

Figure 17: Comparison between $I L$ of reference cases (black) and optimized single wall (red) for $A_{\max }=8$ $\mathrm{m}^{2}$. Dashed curves represent cumulative moving averages $\operatorname{CIL}\left(x_{1}^{\mathrm{r}}\right)$ computed starting from $x_{1}^{\mathrm{r}}=5 \mathrm{~m}$. Dashed vertical lines represent the receiver point used in the optimization process $\left(x_{1}^{\mathrm{r}}=7.5 \mathrm{~m}\right)$.

moving averages computed starting from $x_{1}^{\mathrm{r}}=5 \mathrm{~m}$ :

$$
\operatorname{CIL}\left(x_{1}^{\mathrm{r}}\right)=\frac{1}{x_{1}^{\mathrm{r}}-5} \int_{5}^{x_{1}^{\mathrm{r}}} \operatorname{IL}\left(x_{1}\right) \mathrm{d} x_{1}
$$

As expected after the optimization process, $I L$ peaks appear near the receiver location: $x_{1}^{\mathrm{r}} / \lambda_{\mathrm{R}}=0.8$ for $20 \mathrm{~Hz}, x_{1}^{\mathrm{r}} / \lambda_{\mathrm{R}}=1.5$ for $38 \mathrm{~Hz}, x_{1}^{\mathrm{r}} / \lambda_{\mathrm{R}}=2.5$ for $62 \mathrm{~Hz}$, and $x_{1}^{\mathrm{r}} / \lambda_{\mathrm{R}}=3.2$ for $80 \mathrm{~Hz}$. Also, peaks and valleys appear every Rayleigh wavelength. This localized effect may be representative of what happens along the whole receiver zone or not. Cumulative moving averages $C I L$ become approximately constant beyond $10 \lambda_{\mathrm{R}}$, which agrees to the usual definition of the average insertion loss as $\bar{I} L=C I L\left(10 \lambda_{\mathrm{R}}\right) . \overline{I L}$ shows that optimized single wall barriers perform better than reference cases along the receiver zone in most cases. Therefore, despite obtaining good results in general, it is not possible to assert that these solutions are robust with regard to receiver location.

Sources located at the surface produce both body and surface waves, where the former become less important in comparison with the latter as the observer moves away from the source. Ahmad and Al-Hussaini [18] found that the influence of the source 

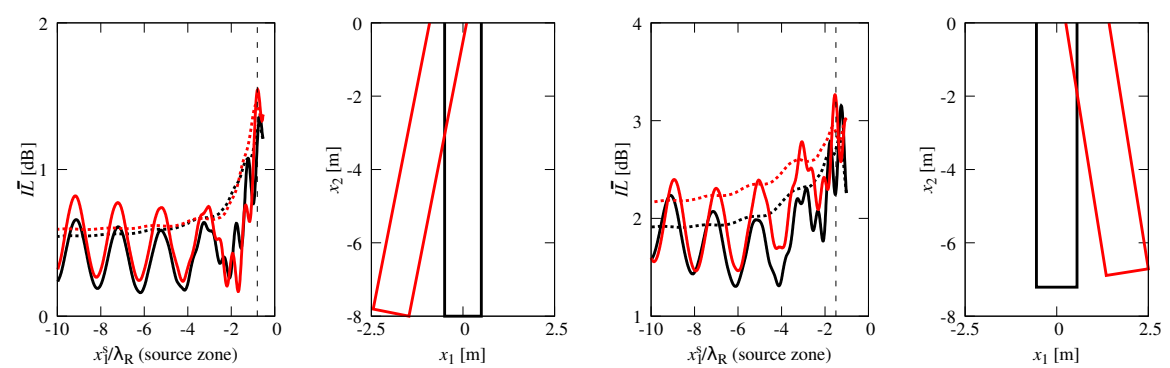

(a) $f^{\text {opt }}=20 \mathrm{~Hz}$
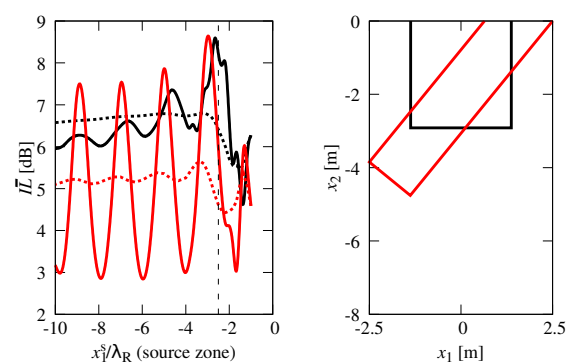

(b) $f^{\text {opt }}=38 \mathrm{~Hz}$

(c) $f^{\text {opt }}=62 \mathrm{~Hz}$
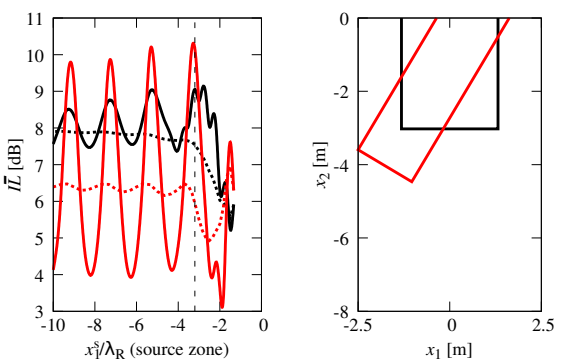

(d) $f^{\text {opt }}=80 \mathrm{~Hz}$

Figure 18: Comparison between $\bar{I} L$ of reference cases (black) and optimized single wall (red) for $A_{\max }=8$ $\mathrm{m}^{2}$. Dashed curves represent cumulative moving averages of $\overline{I L}$ computed starting from $x_{1}^{\mathrm{s}}=-5 \mathrm{~m}$. Dashed vertical lines represent the source point used in the optimization process $\left(x_{1}^{\mathrm{s}}=-7.5 \mathrm{~m}\right)$.

location on the isolation effectiveness is relatively small for sources located beyond three times the Rayleigh wavelength. However, the ratio of source-barrier spacing and Rayleigh wavelength $\left(x_{1} / \lambda_{\mathrm{R}}\right)$ noticeably affects the effectiveness because constructive and destructive patterns from the interaction between source and barrier are altered. For the same representative cases as in Fig. 17, Fig. 18 shows average insertion losses $\overline{I L}$ as the source point is moved away from the barrier, and also their corresponding cumulative moving averages. A highly oscillating $I \bar{L} L$ is observed for $x_{1}^{\mathrm{s}}<4 \lambda_{\mathrm{R}}$ due to the complex interaction of body and surface waves between source and barrier. Beyond $4 \lambda_{R}$, an stable oscillation of $\overline{I L}$ is present because source and barrier interaction is mainly due to surface waves. This oscillation is relatively small for vertical and centered barriers. For inclined barriers, however, these oscillations have a greater amplitude, which reveals that inclined barriers are more sensitive to source location.

All things considered, despite obtaining good designs when varying source and receiver location in some cases, if robustness with regard to these factors is desired, then the optimization problem should be accordingly reformulated. 


\subsection{Optimized double wall barriers}

The second barrier topology is a double wall barrier described by eight design variables $\left(N_{\mathrm{dv}}=8\right)$, see Figure $2 \mathrm{~b}$. The design variables $a_{1}$ to $a_{4}$ are the same as those of the single wall barrier, and associated with the left wall $\left(\Omega_{\mathrm{b} 1}\right)$. Analogously, design variables $a_{5}$ to $a_{8}$ are related to the right wall $\left(\Omega_{\mathrm{b} 2}\right)$. There are eight parametrized geometry points $\left(N_{\mathrm{gp}}=8\right)$, which can be be obtained from:

$$
\begin{aligned}
& \mathbf{p}^{(1)}=\left(a_{1}+\left(a_{4} / 2\right) / \cos \theta_{1}, 0\right), \mathbf{p}^{(2)}=\left(a_{2}+\left(a_{4} / 2\right) \cos \theta_{1}, a_{3}+\left(a_{4} / 2\right) \sin \theta_{1}\right) \\
& \mathbf{p}^{(3)}=\left(a_{2}-\left(a_{4} / 2\right) \cos \theta_{1}, a_{3}-\left(a_{4} / 2\right) \sin \theta_{1}\right), \mathbf{p}^{(4)}=\left(a_{1}-\left(a_{4} / 2\right) / \cos \theta_{1}, 0\right) \\
& \mathbf{p}^{(5)}=\left(a_{5}+\left(a_{8} / 2\right) / \cos \theta_{2}, 0\right), \mathbf{p}^{(6)}=\left(a_{6}+\left(a_{8} / 2\right) \cos \theta_{2}, a_{7}+\left(a_{8} / 2\right) \sin \theta_{2}\right) \\
& \mathbf{p}^{(7)}=\left(a_{6}-\left(a_{8} / 2\right) \cos \theta_{2}, a_{7}-\left(a_{8} / 2\right) \sin \theta_{2}\right), \mathbf{p}^{(8)}=\left(a_{5}-\left(a_{8} / 2\right) / \cos \theta_{2}, 0\right)
\end{aligned}
$$

where $\theta_{1}=\arctan \left(\left(a_{1}-a_{2}\right) / a_{3}\right)$ and $\theta_{2}=\arctan \left(\left(a_{5}-a_{6}\right) / a_{7}\right)$. In order to preserve the topology during the optimization process, compatibility constraints considered in Eq. (12d) must be imposed. The following three constraints $\left(N_{\mathrm{c}}=3\right)$ between points of both walls are defined:

$$
\begin{aligned}
& p_{1}^{(1)}<p_{1}^{(8)} \Rightarrow g_{1}=p_{1}^{(1)}-p_{1}^{(8)}+\epsilon \leq 0 \\
& A^{1,2,7}>0 \Rightarrow g_{2}=-A^{1,2,7}+\epsilon \leq 0 \\
& A^{8,2,7}>0 \Rightarrow g_{3}=-A^{8,2,7}+\epsilon \leq 0
\end{aligned}
$$

where $A^{i, j, k}$ denotes the signed area of the triangle formed by points $\mathbf{p}^{(i)}, \mathbf{p}^{(j)}$, and $\mathbf{p}^{(k)}$, and $\epsilon$ is a small constant that guarantees the original strict inequality. Constraints indicated by Eqs. (19a-19c) guarantee that the quadrilateral formed by points $\mathbf{p}^{(1)}$, $\mathbf{p}^{(2)}, \mathbf{p}^{(7)}$ and $\mathbf{p}^{(8)}$ is convex, thus collision between walls is strictly avoided. For the economic constraint defined in Eq. (12e), the considered area is that of both walls.

The optimized designs for single-frequency sources of 20, 26, 44, 50, 74 and $80 \mathrm{~Hz}$ are shown in Figure 19. Most optimized designs for low and mid frequency sources, i.e. from $f^{\mathrm{opt}}=20$ to $f^{\mathrm{opt}}=50 \mathrm{~Hz}$, share several characteristics with the corresponding optimized single wall barriers (see Figure 12). For the sources of 20 and $26 \mathrm{~Hz}$, the walls on the left hand side have similar angles and positions regardless of $A_{\max }$, and the same is observed for the walls on the right hand side for sources of 44 and $50 \mathrm{~Hz}$. The opposite walls are located at the boundary of the design domain, and are approximately 

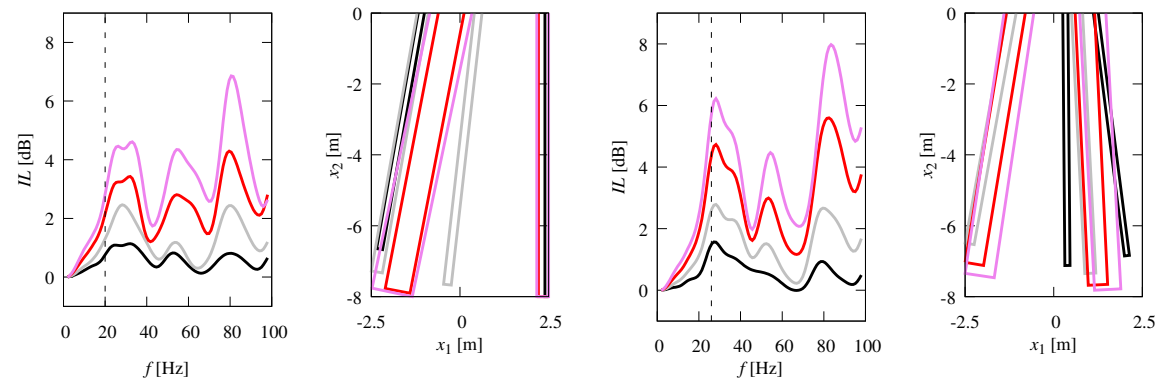

(a) $f^{\text {opt }}=20 \mathrm{~Hz}$
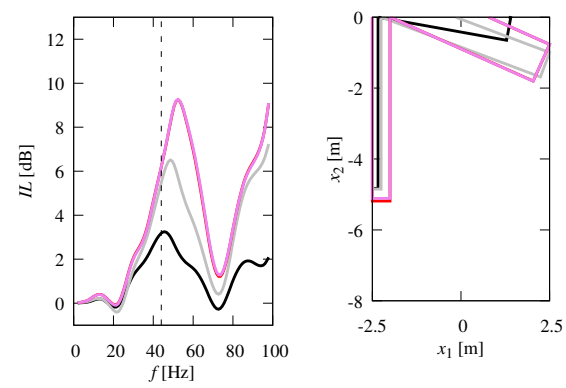

(b) $f^{\text {opt }}=26 \mathrm{~Hz}$

(c) $f^{\text {opt }}=44 \mathrm{~Hz}$
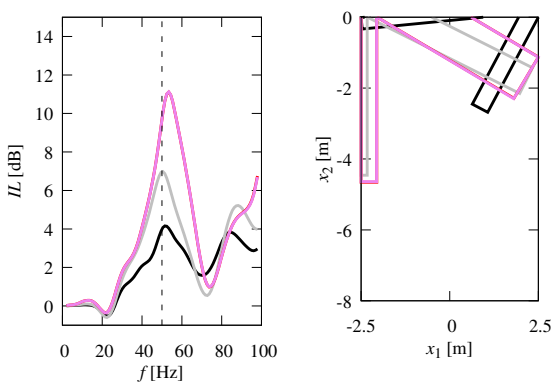

(d) $f^{\mathrm{opt}}=50 \mathrm{~Hz}$
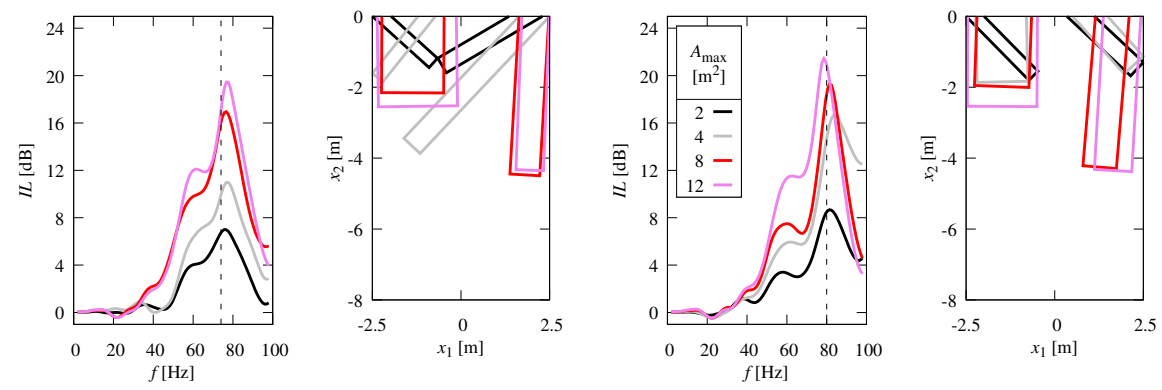

(e) $f^{\text {opt }}=74 \mathrm{~Hz}$

(f) $f^{\text {opt }}=80 \mathrm{~Hz}$

Figure 19: Optimized double wall barriers for single-frequency sources 


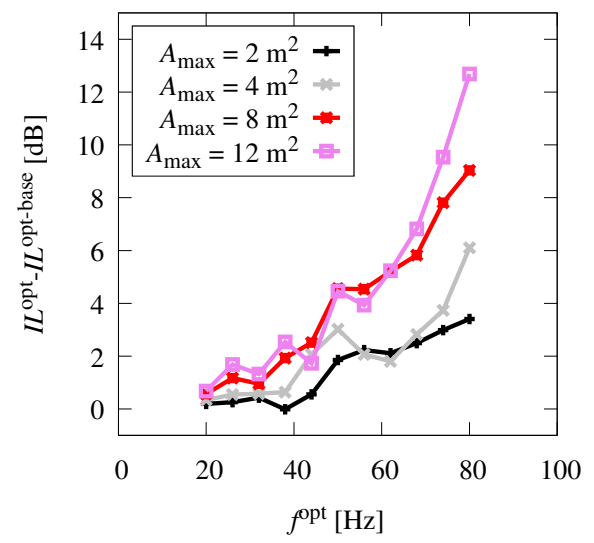

Figure 20: Insertion loss improvement of optimized double wall barriers with respect to optimized reference cases for each target frequency $f^{\text {opt }}$

5 meters deep, i.e. $1.18 \lambda_{\mathrm{R}}$ for $44 \mathrm{~Hz}$ and $1.34 \lambda_{\mathrm{R}}$ for $50 \mathrm{~Hz}$. Note also that the optimized designs for $A_{\max }=12 \mathrm{~m}^{2}$ are similar to those for $A_{\max }=8 \mathrm{~m}^{2}$. For high frequency sources, little correspondence with the optimized single wall barriers is observed. The depths reached by the walls on the left hand side are around 2 meter, i.e. $0.8 \lambda_{\mathrm{R}}$ for 74 $\mathrm{Hz}$ and $0.86 \lambda_{\mathrm{R}}$ for $80 \mathrm{~Hz}$, and they are very thick walls (nearly square blocks), except for a small value of $A_{\max }$. The depths reached by the walls on the right hand side are around 2 meter or 4 meter, and they all are relatively thin walls.

For the objective function associated with single-frequency sources, the improvement of the obtained global minima with respect to the optimized reference cases is shown in Figure 20. It is observed that the improvement is small $(<0.5 \mathrm{~dB})$ for the lowest frequency $(20 \mathrm{~Hz})$, but it increases steadily with the frequency and the available area $A_{\max }$ and reaches remarkably high gains when compared to the optimized reference cases. Unlike the single wall barriers (see Figure 13), which exhibit an nonuniform improvement within the considered range of single-frequency sources, one can always expect that the optimization of double wall barriers would achieve relevant improvement.

Except for a $20 \mathrm{~Hz}$ harmonic source, optimized double wall barriers lead to significant improvements with respect to optimized single wall barriers. In the following paragraphs, we are going to discuss in more detail what is happening physically for the optimized designs for low $(26 \mathrm{~Hz})$, medium $(50 \mathrm{~Hz})$ and high $(74 \mathrm{~Hz})$ frequency sources. 


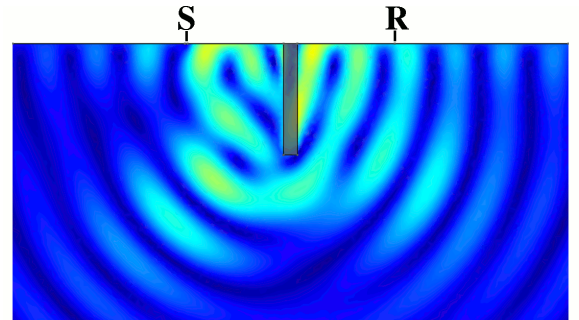

$\mathbf{S}$

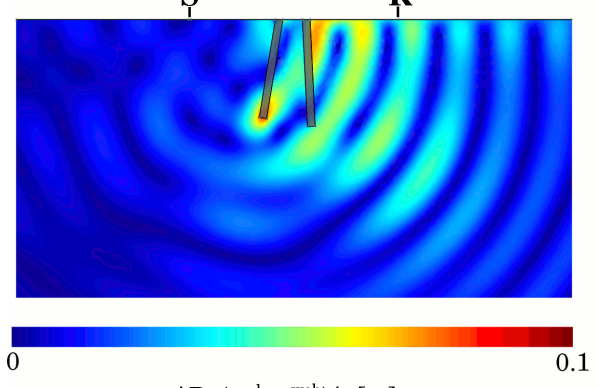

$\left|\operatorname{Re}\left(\mathbf{u}^{\mathrm{wb}}-\mathbf{u}^{\mathrm{wob}}\right)\right|[\mathrm{m}]$
S

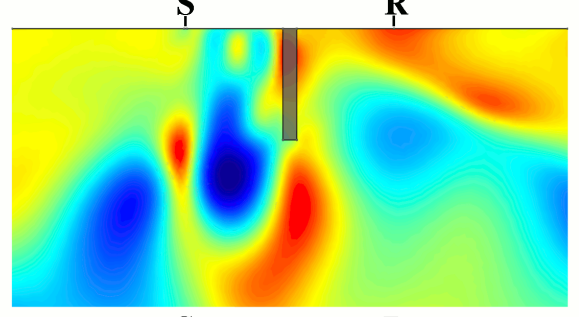

$\mathbf{S}$

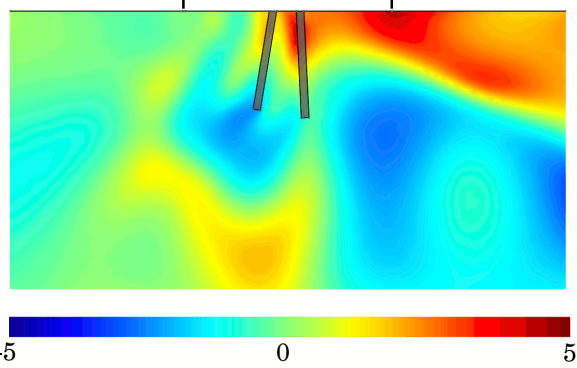

IL $[\mathrm{dB}]$

Figure 21: Comparison between diffracted displacement field and $I L$ color maps of optimized reference case (top) and optimized double wall barrier (bottom) for $f^{\text {opt }}=26 \mathrm{~Hz}$ and $A_{\max }=8 \mathrm{~m}^{2}$

Figure 21 shows a comparison between the optimized reference case and optimized double wall barrier for a single-frequency source of $f^{\text {opt }}=26 \mathrm{~Hz}$. A modest $1.2 \mathrm{~dB}$ improvement is achieved by the optimized double wall barrier $(I L=4.4 \mathrm{~dB})$ when compared to the optimized reference case $(I L=3.2 \mathrm{~dB})$ at the receiver point. The comparison between the displacement fields shows that the optimized double wall barrier relies less on reflection, and instead transmitted waves carry most of the energy towards the inside of the halfspace and away from the receiver. The overall effect is a better insertion loss along the receiver side, and incidentally less insertion gain along the source side.

Figure 22 shows insertion loss maps for optimized double wall barriers for $f^{\text {opt }}=$ $50 \mathrm{~Hz}$ and $f^{\text {opt }}=74 \mathrm{~Hz}$ when the available area is $A_{\max }=8 \mathrm{~m}^{2}$. The insertion loss maps are shown individually for walls on the left and right hand side, and finally the complete double wall barrier. The idea is to observe the contribution of each wall, and their combined effect. For the case $f^{\text {opt }}=50 \mathrm{~Hz}$, the wall on the left hand side acts as a deep wall (depth is $1.25 \lambda_{\mathrm{R}}$ ), and the individual insertion loss at the receiver point is $1.31 \mathrm{~dB}$. The wall on the right hand side is a highly inclined thick wall that partly reflects incoming waves, but also acts as a waveguide taking transmitted waves towards the inside of the soil. The insertion loss of the latter wall alone is $6.15 \mathrm{~dB}$ at 


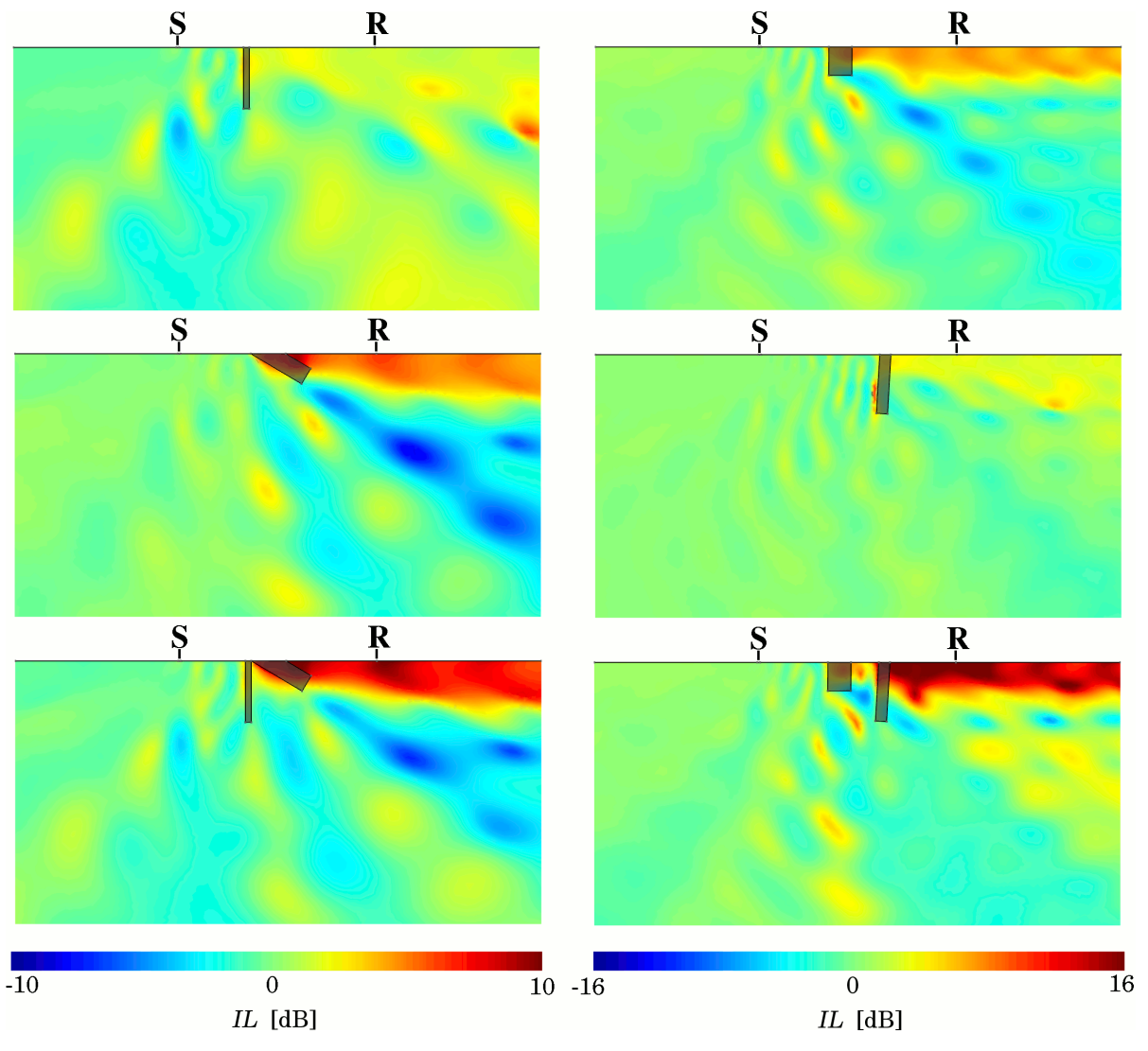

Figure 22: Comparison between $I L$ color maps of optimized double wall barriers for $f^{\text {opt }}=50 \mathrm{~Hz}$ (left) and $f^{\text {opt }}=74 \mathrm{~Hz}$ (right) when $A_{\max }=8 \mathrm{~m}^{2}$. Top: wall on the left hand side. Middle: wall on the right hand side. Bottom: double wall barrier. 
the receiver point. The insertion loss of the double wall barrier is $9.65 \mathrm{~dB}$, showing that both walls complement each other and work in a synergic way. For the case $f^{\text {opt }}=74$ $\mathrm{Hz}$, the wall on the left hand side is a thick wall (depth is $0.86 \lambda_{\mathrm{R}}$ ) which is partly reflecting incoming waves but also producing inclined transmitted waves away from the source. The insertion loss at the receiver point of this wall alone is $6.77 \mathrm{~dB}$. The wall at the right hand side is a very deep wall (depth is $1.78 \lambda_{\mathrm{R}}$ ), and the individual insertion loss at the receiver point is only $2.7 \mathrm{~dB}$. The complete double wall barrier works by partly reflecting incoming waves on the wall on the left hand side, which also produces inclined transmitted waves that are partly reflected on the wall on the right hand side. Both walls work in a very productive manner producing an insertion loss of $15.9 \mathrm{~dB}$, which is much higher than individual contributions.

The obtained global minima for broadband sources and for harmonic sources within a frequency range are respectively depicted in Figs. 23 and 24 together with their insertion loss spectra. As in the case of single wall barriers, optimized designs for broadband sources are very similar to those for high frequency harmonic sources, and optimized barriers for harmonic sources within a frequency range are quite similar to those of low frequency harmonic sources. In the case of optimizing for broadband sources, the improvements with respect to the optimized reference cases (Figure 9) are larger than $1 \mathrm{~dB}$, and increase with the available area reaching up to $3 \mathrm{~dB}$ for $A_{\max }=12$ $\mathrm{m}^{2}$. In this sense, optimized double wall barriers perform better than single walls. When optimizing for harmonic sources within a frequency range, the improvement in performance with respect to the optimized reference cases (Figure 11) hardly reaches $0.5 \mathrm{~dB}$ for all values of $A_{\max }$.

In summary, it has been found that optimized inclined double wall barriers generally improve the performance of single wall barriers with the same maximum total area. This improvement is, however, insignificant when optimizing for sources whose relevant frequencies lead to Rayleigh wavelengths similar to the depth of the design domain, but it increases for higher frequencies. This conclusion contrasts with that from [14], where only small improvements are found for vertical double and single walls.

\section{Conclusions}

In this paper, we have examined the possibilities of shape optimization of two wave barrier topologies: a single wall barrier, and a double wall barrier. In the former case, 

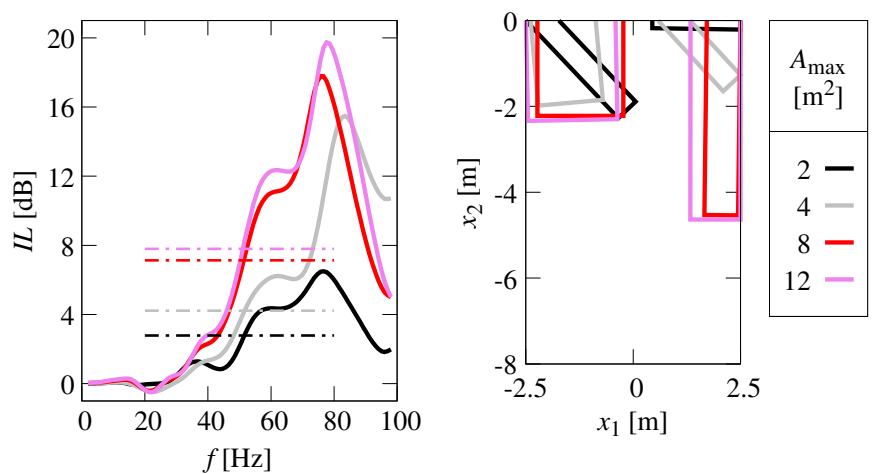

Figure 23: Optimized double wall barriers for broadband sources $\left(f^{\mathrm{opt}}=[20,80] \mathrm{Hz}\right)$
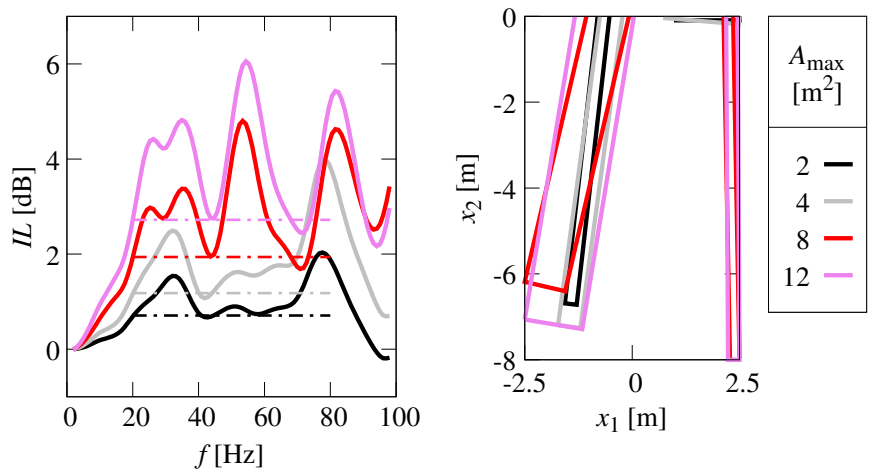

Figure 24: Optimized double wall barriers for harmonic sources within a frequency range $\left(f^{\text {opt }}=[20,80]\right.$ $\mathrm{Hz})$ 
we have also studied the particular case of a vertical and centered wall, which has been taken as optimized reference case. Despite the relative simplicity of these topologies, they have the potential for improving conventional designs. Gradient-based optimization is used, where a pure boundary element formulation supplies the objective function and its gradient. Since point source and receiver are assumed, solutions are not robust with respect their location. However, most of the obtained optimized designs also improve reference cases in terms of average insertion loss along a receiver zone.

When the depth of the design domain is limited up to approximately one Rayleigh wavelength, the optimization of the studied barrier topologies does not lead to significant improvements. Once the depth of the design domain is greater than $1.1 \lambda_{\mathrm{R}}(f>26$ $\mathrm{Hz}$ in our case study), relevant room for optimization is observed, especially for double wall barriers. More detailed conclusions can be drawn from this work:

Single-frequency sources.

- For the simplest case of a vertical and centered single wall barrier, it is observed that a wall depth of $\lambda_{\mathrm{R}}$ (and width $A_{\max } / \lambda_{\mathrm{R}}$ ) is far from optimal. Optimized designs fall into one of three types of barriers: a) deep walls, b) slabs, or c) thick walls of nearly unitary aspect ratio. Each one of these may become optimal depending on the frequency and the available material $\left(A_{\max }\right)$.

- For general single wall barriers, little room for optimization is found in the low frequency range ( $f<38 \mathrm{~Hz}$ in our case study). For higher frequencies, improvements of 1 to $4 \mathrm{~dB}$ with respect to optimized reference cases can be achieved. However, such gains are not reached over the entire frequency range.

- For double wall barriers, small improvements with respect to optimized reference cases are found at low frequencies. However, they increase steadily with the frequency and the available area, reaching quite remarkable gains between 2 and $13 \mathrm{~dB}$.

Broadband sources. Designs very similar to those of the highest frequency are consistently obtained. This result is reasonable because the objective function is a simple average insertion loss within a frequency range, and reduction is more easily obtained at the high frequency range. Therefore, it is appropriate to use as initial points the local minima obtained from the optimization of single-frequency sources at the highest frequencies of broadband sources. 
Harmonic sources within a frequency range. Designs very similar to those of the lowest frequency are obtained. Analogously to the optimization for broadband sources, this observation can be used to establish better initial points.

\section{Acknowledgements}

J.D.R. Bordón was recipient of the research fellowship FPU13/01224 and research short stay grant EST14/00437, both from the Ministerio de Educación, Cultura y Deportes of Spain. C. Van hoorickx was a doctoral fellow of Research Foundation Flanders (FWO) (grant number 11U4616N).

The authors affiliated to University of Las Palmas de Gran Canaria are grateful for the support from the Subdirección General de Proyectos de Investigación of the Ministerio de Economía y Competitividad (MINECO) of Spain and FEDER through Research Projects BIA2014-57640-R and BIA2017-88770-R.

The authors affiliated to KU Leuven gratefully acknowledge the support from the Research Council of KU Leuven through the funding of the project C16/17/008 Efficient methods for large-scale PDE-constrained optimization in the presence of uncertainty and complex technological constraints.

\section{References}

[1] H. Mercier W. J. Ammann, F. Deischl, J. Eisenmann, I. Floegl, G. H. Hirsch, G. K. Klein, G. J. Lande, O. Mahrenholtz, H. G. Natke, H. Nussbaumer, A. J. Pretlove, J. H. Rainer, E.-U. Saemann, and L. Steinbeisser. Vibration Problems in Structures. Birkhäuser Basel, 1995.

[2] International Organization for Standardization. ISO 2631-2:2003 Mechanical vibration and shock - Evaluation of human exposure to whole-body vibration - Part 2: Vibration in buildings ( $1 \mathrm{~Hz}$ to $80 \mathrm{~Hz})$.

[3] International Organization for Standardization. ISO 14837-1:2005 Mechanical vibration - Ground-borne noise and vibration arising from rail systems - Part 1: General guidance.

[4] R.D. Woods. Screening of surface waves in soils. Journal of the Soil Mechanics and Foundation Division, Proceedings of the ASCE, 94(SM4):951-979, 1968. 
[5] P. H. Tsai and T. S. Chang. Effects of open trench siding on vibration-screening effectiveness using the two-dimensional boundary element method. Soil Dynamics and Earthquake Engineering, 29:865-873, 2009.

[6] D.J. Thompson, J. Jiang, M.G.R. Toward, M.F.M. Hussein, E. Ntotsios, A. Dijckmans, P. Coulier, G. Lombaert, and G. Degrande. Reducing railway-induced ground-borne vibration by using open trenches and soft-filled barriers. Soil Dynamics and Earthquake Engineering, 88(Supplement C):45-59, 2016.

[7] L. Andersen and S. R. K. Nielsen. Reduction of ground vibration by means of barriers or soil improvement along a railway track. Soil Dynamics and Earthquake Engineering, 25:701-716, 2005.

[8] P. Coulier, V. Cuéllar, G. Degrande, and G. Lombaert. Experimental and numerical evaluation of the effectiveness of a stiff wave barrier in the soil. Soil Dynamics and Earthquake Engineering, 77(Supplement C):238-253, 2015.

[9] A. Dijckmans, A. Ekblad, A. Smekal, G. Degrande, and G. Lombaert. Efficacy of a sheet pile wall as a wave barrier for railway induced ground vibration. Soil Dynamics and Earthquake Engineering, 84(Supplement C):55-69, 2016.

[10] S. E. Kattis, D. Polyzos, and D. E. Beskos. Vibration isolation by a row of piles using a 3-D frequency domain BEM. International Journal for Numerical Methods in Engineering, 46:713-728, 1999.

[11] A. Colombi, S. Guenneau, P. Roux, and R. V. Craster. Transformation seismology: composite soil lenses for steering surface elastic rayleigh waves. Scientific Reports, 6(25320), 2016.

[12] C. Van hoorickx, O. Sigmund, M. Schevenels, B. S. Lazarov, and G. Lombaert. Topology optimization of two-dimensional elastic wave barriers. Journal of Sound and Vibration, 376:95-111, 2016.

[13] M. Bendsøe and O. Sigmund. Topology Optimization: Theory, Methods and Applications. Springer, Berlin, 2003.

[14] C. Van hoorickx, M. Schevenels, and G. Lombaert. Double wall barriers for the reduction of ground vibration transmission. Soil Dynamics and Earthquake Engineering, 97:1-13, 2017. 
[15] L. Andersen and M. Liingaard. Vibration screening with sheet pile walls. In Takemiya, editor, Environmental Vibrations: Prediction, Monitoring and Evaluation (ISEV 2005), 2005.

[16] L. Andersen and A. J. Augustesen. Mitigation of traffic-induced ground vibration by inclined wave barriers - a three-dimensional numerical analysis. In M. Pawelczyk and D. Bismor, editors, Recent developments in Acoustics, Noise and Vibration: Proceedings of the 16th International Congress on Sound and Vibration (ICSV16). International Institute of Acoustics and Vibration (IIAV), 2009.

[17] D. E. Beskos, B. Dasgupta, and I. G. Vardoulakis. Vibration isolation using open or filled trenches. Part 1: 2-D homogeneous soil. Computational Mechanics, $1: 43-63,1986$.

[18] S. Ahmad and T. M. Al-Hussaini. Simplified design for vibration screening by open and in-filled trenches. Journal of Geotechnical Engineering, 117:67-88, 1991.

[19] P. K. Banerjee, S. Ahmad, and K. Chen. Advanced application of BEM to wave barriers in multi-layered three-dimensional soil media. Earthquake Engineering and Structural Dynamics, 16:1041-1060, 1988.

[20] B. Dasgupta, D. E. Beskos, and I. G. Vardoulakis. Vibration isolation using open or filled trenches. Part 2: 3-D homogeneous soil. Computational Mechanics, 6:129-142, 1990.

[21] J. D. R. Bordón, J. J. Aznárez, and O. Maeso. Two-dimensional numerical approach for the vibration isolation analysis of thin walled wave barriers in poroelastic soils. Computers and Geotechnics, 71:168-179, 2016.

[22] L. Andersen and C. J. C. Jones. Coupled boundary and finite element analysis of vibration from railway tunnels: a comparison of two- and three-dimensional models. Journal of Sound and Vibration, 293:611-625, 2006.

[23] M. R. Barone and D. A. Caulk. Optimal arrangement of holes in a twodimensional heat conductor by a special boundary integral method. International Journal for Numerical Methods in Engineering, 18:675-685, 1982. 
[24] R. A. Meric. Boundary integral equation and conjugate gradient methods for optimal boundary heating of solids. International Journal of Heat and Mass Transfer, 26:261-268, 1983.

[25] C.A. Mota Soares, H. C. Rodrigues, and K. K. Choi. Shape optimal structural design using boundary elements and minimum compliance techniques. Journal of Mechanisms, Transmissions, and Automation in Design, 106:518-523, 1984.

[26] R. A. Meric. Optimal loading of solids by the boundary element method. International Journal of Engineering Science, 23:1101-1111, 1985.

[27] M. Bonnet. BIE and material differentiation applied to the formulation of obstacle inverse problems. Engineering Analysis with Boundary Elements, 15:121-136, 1995.

[28] M. Bonnet. Regularized BIE formulations for first- and second-order shape sensitivity of elastic fields. Computers $\mathcal{E}$ Structures, 56:799-811, 1995.

[29] M. Bonnet. Boundary element based formulations for crack shape sensitivity analysis. Engineering Analysis with Boundary Elements, 25:347-362, 2001.

[30] M. Bonnet, T. Burczynski, and M. Nowakowski. Sensitivity analysis for shape perturbation of cavity or internal crack using BIE and adjoint variable approach. International Journal of Solids and Structures, 39:2365-2385, 2002.

[31] M. Bonnet. Differentiability of strongly singular and hypersingular boundary integral formulations with respect to boundary perturbations. Computational Mechanics, 19:240-246, 1997.

[32] R. Gallego and J. Suárez. Solution of inverse problems by boundary integral equations without residual minimization. International Journal of Solids and Structures, 37:5629-5652, 2000.

[33] R. Gallego and J. Suárez. Numerical solution of the variation boundary integral equation for inverse problems. International Journal for Numerical Methods in Engineering, 49:501-518, 2000.

[34] G. Rus. Numerical methods for nondestructive identification of defects. $\mathrm{PhD}$ thesis, University of Granada, 2001. 
[35] G. Rus and R. Gallego. Boundary integral equation for inclusion and cavity shape sensitivity in harmonic elastodynamics. Engineering Analysis with Boundary Elements, 29:77-91, 2005.

[36] G. Rus and R. Gallego. Hypersingular shape sensitivity boundary integral equation for crack identification under harmonic elastodynamic excitation. Computational Methods in Applied Mechanics and Engineering, 196:2596-2618, 2007.

[37] J. Domínguez. Boundary Elements in Dynamics. International Series on Computational Engineering. Computational Mechanics Publications, 1993.

[38] F. Hartmann. The Somigliana identity on piecewise smooth surfaces. Journal of Elasticity, 11(4):403-423, 1981.

[39] S. Saigal, R. Aithal, and J. H. Kane. Semianalytical structural sensitivity formulation in boundary elements. American Institute of Aeronautics and Astronautics, 27(11):1615-1621, 1989.

[40] C. Geuzaine and J. F Remacle. Gmsh: a three-dimensional finite element mesh generator with built-in pre- and post-processing facilities. International Journal for Numerical Methods in Engineering, 79(11):1309-1331, 2009.

[41] T. W. Sederberg. Free-form deformation of solid geometric models. In Proceeding of the 13th annual conference on Computer graphics and Interactive techniques, volume 20, pages 151-160, Dallas, August 18-22 1986.

[42] K. K. Choi and K.-H. Chang. A study of design velocity field computation for shape optimal design. Finite Elements in Analysis and Design, 15:317-341, 1994.

[43] MATLAB. version 8.3.0 (R2014a). The MathWorks Inc., 2014.

[44] A. H. G. Rinnooy Kan and G. T. Timmer. Stochastic global optimization methods. Part I: clustering methods. Mathematical Programming, 39:27-56, 1987. 